\title{
High USP6NL levels in breast cancer sustain chronic AKT phosphorylation and GLUT1 stability fueling aerobic glycolysis
}

Daniele Avanzato ${ }^{1,2}$, Emanuela Pupo ${ }^{1,2}$, Nadia Ducano ${ }^{2}$ Claudio Isella ${ }^{2}$, Giovanni Bertalot $^{3}$, Chiara Luise $^{3}$, Salvatore Pece ${ }^{3,4}$, Alejandra Bruna ${ }^{5}$, Oscar M Rueda ${ }^{5}$, Carlos Caldas $^{5}$, Pier Paolo Di Fiore ${ }^{3,4,6}$, Anna Sapino ${ }^{2,7}$ and Letizia Lanzetti ${ }^{1,2}$

${ }^{1}$ Dept. of Oncology, University of Torino Medical School, Torino, Italy;

${ }^{2}$ Candiolo Cancer Institute, FPO - IRCCS, Candiolo, Torino, Italy;

${ }^{3}$ Molecular Medicine Program, European Institute of Oncology, Milan, Italy

${ }^{4}$ DIPO, Dept. of Oncology and Hemato-Oncology, University of Milan, Milan, Italy;

${ }^{5}$ Dept. of Oncology and Cancer Research UK Cambridge Institute, Li Ka Shing Centre, University of Cambridge, Cambridge, UK;

${ }^{6}$ IFOM, The FIRC Institute for Molecular Oncology Foundation, Milan, Italy;

${ }^{7}$ Dept. of Medical Sciences, University of Torino Medical School, Torino, Italy.

Running title: "USP6NL fuels aerobic glycolysis in breast cancer"

Keywords: USP6NL, RN-tre, breast cancer, GLUT1, aerobic glycolysis

Correspondence should be addressed to:

Letizia Lanzetti

Candiolo Cancer Institute

Str. Provinciale 142, 10060 Candiolo,

Turin Italy

Tel. +390119933255

Fax +390119933524

e-mail: letizia.lanzetti@ircc.it 


\section{Financial support}

Work in the authors' lab is supported by grants from the Associazione Italiana per la Ricerca sul Cancro (AIRC Investigator Grant, project 15180 to L.L. and 14404, MCO 10.000 and extension to PPDF, 5xmille MCO Extension to AS), Fondo Ricerca Locale 2017 (University of Turin) to LL, MIUR (the Italian Ministry of University and Scientific Research) to PPDF, the Monzino Foundation to PPDF, FPRC 5xmille Ministero Salute 2011, 2014 to AS and 2015 to LL, and Cancer UK to A.B., O.M.R. and C.C.

\section{Conflict of interest}

The authors declare no potential conflicts of interest.

\section{Word count 5224}

\section{Number of Figures 7}




\section{ABSTRACT}

USP6NL, also named RN-tre, is a GTPase activating protein (GAP) involved in control of endocytosis and signal transduction. Here we report that USP6NL is overexpressed in breast cancer (BC), mainly of the basal-like/integrative cluster 10 subtype. Increased USP6NL levels were accompanied by gene amplification and were associated with worse prognosis in the METABRIC dataset, retaining prognostic value in multivariable analysis. High levels of USP6NL in BC cells delayed endocytosis and degradation of the epidermal growth factor receptor (EGFR), causing chronic AKT activation. In turn, AKT stabilized the glucose transporter GLUT1 at the plasma membrane, increasing aerobic glycolysis. In agreement, elevated USP6NL sensitized BC cells to glucose deprivation, indicating that their glycolytic capacity relies on this protein. Depletion of USP6NL accelerated EGFR/AKT downregulation and GLUT1 degradation, impairing cell proliferation exclusively in BC cells that harbored increased levels of USP6NL. Overall, these findings argue that USP6NL overexpression generates a metabolic rewiring that is essential to foster the glycolytic demand of $\mathrm{BC}$ cells and promote their proliferation. 


\section{INTRODUCTION}

A large fraction of BCs, especially of the basal-like subtype, display unique gene ontologies of growth factor signaling, glycolysis and gluconeogenesis (1) and are characterized by increased glycolysis and dependence on this metabolic drift for proliferation $(2,3)$.

High rates of glucose uptake are achieved through transcriptional up regulation and increased targeting of GLUT1, a member of the glucose transporter family, to the plasma membrane (PM) (4). Localization of GLUT1 to the cell surface is largely regulated through its endocytic traffic (5-11), controlled, in non-transformed cells, by cytokine stimulation $(12,13)$ or energy stress $(6)$. In cancer cells, several oncogenic lesions, including hyper activation of the EGFR/PI3K/AKT signaling pathway, induce accumulation of GLUT1 at the cell surface resulting in increased aerobic glycolysis (14-16). However, the molecular determinants of this signaling-metabolic rewiring are largely unknown.

USP6NL is GAP protein that stimulates GTP hydrolysis on various members of the Rab family, thereby inhibiting endocytosis of PM receptors and retrograde Golgi transport (17-20). We found that USP6NL is amplified and overexpressed in a subset of BCs, mainly belonging to the basal-like Integrative Cluster 10 subtype. USP6NL overexpression correlates with worse prognosis suggesting a role in the natural history of the tumor. This is strengthened by data showing that functional ablation of USP6NL inhibits proliferation and glycolysis exclusively in BC cells harboring high levels of this protein.

Our findings argue, therefore, that USP6NL is a regulator of the crosstalk between signaling and metabolic pathways whose elevation causes glycolysis addiction in BC cells. 


\section{MATERIALS AND METHODS}

\section{Cell cultures, silencing and constructs}

HCC70, HCC1187, H1299 (ATCC) were grown in RPMI (Sigma) with 10\% FBS (Euroclone) and 1\% glutamine. MCF10A (a gift of J.S. Brugge, Harvard Medical School, Boston, USA) were grown in DMEM/F12 with EGF $20 \mathrm{ng} / \mathrm{ml}$ (Peprotech), 5\% Horse Serum, Hydrocortisone $0.5 \mathrm{mg} / \mathrm{ml}$, Cholera Toxin $100 \mathrm{ng} / \mathrm{ml}$ and Insulin 10 $\mu \mathrm{g} / \mathrm{ml}$ (Sigma). HMEC (hTERT-HME1) were from ATCC and grown in DMEM/F12 with EGF $20 \mathrm{ng} / \mathrm{ml}$, Hydrocortisone $0.5 \mathrm{mg} / \mathrm{ml}$, Insulin $10 \mu \mathrm{g} / \mathrm{ml}$ and $10 \%$ FBS. The genetic identity of the cell lines was confirmed by STR profiling (Cell ID, Promega), lastly repeated in July 2017. Cells were periodically tested for mycoplasma with Venor GM kit (Minerva biolabs).

For functional ablation experiments, equal number of cells were plated in presence of siRNA oligos, and a second round of transfection was repeated the day after on the adherent cells. Cells were harvested 48 hours later for biochemical experiments or counted for the "proliferation" experiments (see "cell counts", below). Ablation of USP6NL was initially performed with three different oligos (Silencer Select oligos, Ambion, 50 pmoles; negative control: siRNA\#2) using Lipofectamine RNAiMAX (Invitrogen). Since the three oligos yielded comparable results, oligo s18721 alone was subsequently employed, after confirming its specificity in rescue experiments.

Stable, inducible HCC70 and HMEC cell populations were generated by infection with tetracycline-inducible lentiviral vectors (pSLIK) carrying HA-tagged USP6NL or HA-USP6NLR150 (R150) (17,19). In these constructs, the sequence targeted by oligo s18721 was mutated with the Phusion Site-Directed Mutagenesis Kit (Thermo 
Scientific) in a silencing-resistant version: 5'-TAGACAGTATAATCACGCA-3'. Stable populations, including MCF10A-pSLIK-RAB5A (a gift from Giorgio Scita) (21), were established by neomycin selection and grown in media supplemented with $10 \%$ Tet system-approved FBS (Clontech). Expression of USP6NL, R150 or RAB5 was induced by doxycycline $(1 \mu \mathrm{g} / \mathrm{ml}$, Sigma) for 48 hours. HCC70 cells stably expressing PI3KCA were generated by infection with the retroviral construct pBABE puro Myr PIK3CA, a gift from Jean Zhao (Addgene plasmid \# 12523). pLPCX-EGFPmyc-GLUT1 (22) was kindly provided by Jeffrey Pessin and transiently transfected using the X-tremeGENE reagent (Sigma).

\section{Antibodies and reagents}

Antibodies were: affinity-purified rabbit polyclonal anti-USP6NL home made; antiAKT (2920), anti-phosphoAKT-Ser473 (4060), anti-phosphoAKT-Thr308 (2965), antiphosphoGSK3 $\beta$-Ser9 (9336), anti-phosphoEGFR (3777), anti-phosphoMET (3077), Cell Signaling Technology; anti-GSK3 $\beta$ (E-11 sc377213), anti-HA (Y-11 sc-805; F-7 sc-7392), anti-HSP90 (sc-13119), anti-EEA1 (N-19 sc-6415), anti-EGFR (1005 sc03), anti-Rab5 (D-11 sc46692), anti-MET (D-4 sc514148) Santa Cruz; anti-GLUT1 (ab15309), anti-GAPDH (6C5 ab8245), anti-myc (9E10 ab32) AbCam; anti-EGFR (LA22) Millipore. Alexa Fluor Phalloidin 647 (A22287) was from Thermo Scientific. EGF and HGF were from R\&D, MK2206 was from Selleckchem, bafilomycin from Sigma and MG132 from Calbiochem.

\section{Immunohistochemistry}

Immunohistochemistry (IHC) was performed on a tissue microarray (TMA) of 1608 consecutive BCs and 99 normal samples from the European Institute of Oncology in 
Milan, Italy (years 1997-2000). Cases were annotated for clinical and pathological parameters and censored at 10 years from surgery. All tissues were collected via standard operating procedures approved by the Institutional Ethical Board, and informed consent was obtained for all tissue specimens linked with clinical data.

TMAs were stained in a Bond Max Automated IHC Vision Biosystem (Leica Microsystems) using the Bond Polymer Refine Detection Kit (DS9800). 3- $\mu$ m-thick sections were prepared from formalin-fixed paraffin-embedded blocks, deparaffinized, pre-treated with the Epitope Retrieval Solution $1(\mathrm{pH} 6)$ at $100^{\circ} \mathrm{C}$ for 20 min and then incubated for 30 min with anti-USP6NL (Assay Biotech C19466), 1:100 diluted in Bond Primary Antibody Diluent (AR9352) at final concentration of 10 $\mu \mathrm{g} / \mathrm{ml}$. Slides were acquired with the Aperio ScanScope system (Leica Microsystems).

\section{METABRIC analysis and statistics}

Sample extraction, processing and analysis of the METABRIC samples are described in (23). Differential expression was determined using the limma package (24) followed by survival distributions using the log rank test. Comparison between expression in the Integrative Cluster 10 and the rest of the METABRIC tumors was by two-sided t-test. Survival analysis was by Kaplan-Meier estimates; associations with diseases specific survival was determined by the log rank test for single factors or Cox multiple regression for multiple several factors. The Cox model was stratified by hospital and included tumor size, tumor grade, number of lymph nodes as numeric and the USP6NL copy number aberration as a binary variable. The increasing trend between copy number and expression was determined using the one-sided Jonckheere-Terpstra test from the $\mathrm{R}$ clinfun package. Statistical analysis 
in the cell biology experiments was performed with the non-parametric Wilcoxon test, unpaired data significant levels $<0.02$.

\section{Biochemical assays and Mesoscale analysis}

Total cell lysates were obtained with hot $\left(95^{\circ} \mathrm{C}\right)$ lysis buffer $(125 \mathrm{mM}$ Tris $\mathrm{HCl} \mathrm{pH} 6.8$, 2.5\% SDS), separated on SDS-PAGE gradient gels (Invitrogen) and transferred to nitrocellulose membrane. Densitometry of immunoblots was performed using ImageJ (https://rsb.info.nih.gov/ij/): phospho-AKT and phospho-GSK3 $\beta$ signal were divided by the intensity of the corresponding total protein. GLUT1, phospho-EGFR, EGFR, phospho-MET and MET were normalized over HSP90 or GAPDH.

Multiplex phosphoproteomic analyses were as in (25) with commercially available plates [MAP kinase multiplex assay (K15101D) and phosphoSer(473)AKT/Total (K15100D)] MesoScale Discovery. Phospho-proteins signals, normalized over control, were converted in logarithmic form and represented with heat maps generated by the Gedas program. Measurements of endocytosis of surface biotinylated receptors with sulfo-NHS-SS-biotin (ThermoScientific) and assessment of surface GLUT1 with non cleavable biotin were performed by capture ELISA as in (25).

\section{Immunofluorescence and quantifications}

Immunofluorescence was performed as previously described (26). Primary antibodies were revealed by Alexa Fluor 555- and 488- conjugated antibodies. In the GLUT1/EEA1 staining, permeabilization was in PBS $2 \%$ BSA, $0.1 \%$ saponin for 30 min at RT. Incubation with primary and secondary antibodies was in PBS $0.2 \%$ BSA, $0.1 \%$ saponin. In the phospho-AKT/AKT total stainings, blocking and 
permeabilization was in PBS 5\% donkey serum, 0.3\% Triton-X100. Primary and secondary antibodies were diluted in PBS $0.3 \%$ Triton-X100. In the phospho-EGFR/ EGFR total stainings, blocking and permeabilization were in PBS $2 \%$ BSA, $0.02 \%$ saponin for 10 min at RT. Confocal analysis was performed with a Leica SP8 AOBS microscope. Colocalization between GLUT1 and EEA1 was measured with JacoP plugin of ImageJ. Because variability was recorded among experiments in total-AKT staining, the intensity of phospho-AKT was measured as the Pearson's correlation coefficient between phospho-AKT and Phalloidin provided by the LasAF software (Leica). Measurements of myc-GLUT1 endocytosis are detailed in figure legend.

\section{Cell count, glucose uptake and lactate production}

Cells were counted by Trypan blue exclusion. Briefly, cells silenced or treated as indicated, were collected both from the medium and by trypsinizing the adherent ones. This was necessary since some of the BC cell lines growth either partially in suspension (HCC1187) or detach easily from adherence. Cells were resuspended in complete medium, diluted in Trypan blue solution and counted with a Bürker chamber. At least eight fields/sample were counted in multiple replicates. In the glucose deprivation assays, cells were starved in DMEM 10\% FBS without Dglucose and sodium pyruvate (A14430 Gibco).

Cell toxicity and ATP cell content were evaluated with the CellTox Green Citotoxicity Assay and CellTiter-Glo (Promega).

Glucose uptake was measured with the Glucose Uptake-Glo Assay (Promega). Cells were incubated for 10 minutes in glucose-deprived medium supplemented with $1 \mathrm{mM}$ 2-Deoxy-D-Glucose (2DG). Glucose uptake (fmol/cell/min) was calculated as follows: 
Rate of glucose uptake $=\left([2 \mathrm{DG} 6 \mathrm{P}]{ }^{*}(\right.$ volume of sample $\left.)\right) /\left((\right.$ number of cells $){ }^{*}($ time of uptake)).

Intracellular Lactate production was determined with the fluorimetric L-Lactate Assay Kit (Cayman). The same number of HCC70 and HCC1187 silenced cells were processed and the concentration $(\mu \mathrm{M})$ of L-Lactate was determined as follows:

L-Lactate $(\mu \mathrm{M})=\left[\frac{\mathrm{CF}-(\mathrm{y} \text {-intercept })}{\text { Slope }}\right] * 2 *$ sample dilution

CF is the corrected fluorescence (background subtracted), and slope and intercept were calculated from the standard reference curve. 


\section{RESULTS}

\section{USP6NL is overexpressed in aggressive BCs}

We used the cBioPortal $(27,28)$ to survey alterations affecting USP6NL in BC datasets. In the METABRIC cohort $(23,29)$, high mRNA and gene amplification of USP6NL were detected in $\sim 7 \%$ and $\sim 3.5 \%$, respectively (Figure $1 \mathrm{~A}$ left). Around $75 \%$ of all amplified cases also displayed mRNA up-regulation (Figure 1A left). Moreover, BCs with gains and copy number amplifications showed a proportional increase in USP6NL mRNA expression (Figure 1B). Similar results were obtained from the TCGA dataset (30) (Figure 1A right).

In the METABRIC cohort, expression of USP6NL was higher in the PAM50 Basal and Her2 subtypes vs. Luminal and Normal subtypes $(\mathrm{p}<0.0001)$ (Figure 1C). Using the genomic-based Integrative Cluster classification of BCs (31), we found that USP6NL expression was associated with the Integrative Cluster 10 (IntClust10, Supplementary Figure S1) which incorporates mostly triple-negative tumors and represents a high risk group.

In univariate analysis USP6NL copy number and expression was significantly associated with worse BC specific survival (HR 1.81; $p<0.0001)$ (Figure 1D). This association remained significant in multivariable analysis $\left(H R^{*} 1.46 ; p<0.004\right)$ when grade, size and lymph nodes involvement were considered (Supplementary Table 1).

We investigated the expression of USP6NL in BCs also at the protein level by IHC on TMAs (Methods and Figure 1E). The normal mammary gland displayed heterogeneous staining of USP6NL, with levels comparatively higher in the luminal than in the basal layer (Figure 1E). USP6NL protein expression was, on average, higher in BC samples $(p<0.0001)$. Strongest positivity (score 3 ) was found in $7 \%$ of BCs and never detected in normal samples (Figure 1F), concordant with the up- 
regulation at the mRNA level (Figure 1A). Also in this cohort, strong USP6NL expression correlated with aggressiveness (higher grade and increased percentage of Ki-67 positive cells) and with the molecular subtype of triple-negative BCs (Supplementary Table 2). In the IHC series we did not detect correlation with prognostic outcome likely because of a lower number of events in the USP6NL-high BCs in this cohort vs. the METABRIC one.

Altogether, these results suggest that $U S P 6 N L$ is a copy number driver gene in BCs.

\section{Overexpression of USP6NL in BC cells promotes proliferation and sustains}

\section{AKT phosphorylation in a GAP-dependent manner}

To investigate the role of USP6NL elevation in BCs, we selected two USP6NL-high basal-like BC cell lines expressing high amount of the USP6NL protein, HCC70 and HCC1187, and two non-transformed, USP6NL-low, mammary cell lines, MCF10A and HMEC (Figure 2A). Silencing of USP6NL reduced proliferation in both the USP6NL-high lines, leaving the USP6NL-low cells unaffected (Figure 2A-B and Supplementary Figure S2A-B), arguing that USP6NL-high BC cells depend on this molecule for their optimal growth. The effects of the functional ablation of USP6NL on cell number were due to a decrease in the proliferation rate and not to increased cell death, as it will be subsequently shown.

USP6NL has been found to restrain EGFR endocytosis (17-19). Endocytosis and trafficking control the magnitude of the EGFR signaling (32), suggesting that USP6NL might affect proliferation by dampening intracellular signaling pathways. Thus, we analyzed alterations in the phosphorylation status of key signal transducers: AKT, ERK1/2, JNK and p38, by the MesoScale platform. USP6NL 
depletion significantly reduced AKT phosphorylation in HCC70 and HCC1187 cells, but not in MCF10A (Figure 2C and Supplementary Figures S2B and S3A). Lesser changes in ERK1/2, JNK and p38 phosphorylation were also detected, albeit frequently not significant and not shared between the USP6NL-overexpressing BC cells (Figure 2C).

To validate the specificity of the effects, we performed rescue experiments. We generated stable inducible cell populations by infecting HCC70 cells with lentiviral vectors carrying the silencing-resistant version of USP6NL or its GAP-defective mutant R150 (19). Re-expression of the wild type protein in USP6NL-silenced cells rescued AKT activation and cell proliferation (Figure 2D-E). Conversely, reexpression of R150 did not recover any of the phenotypes, revealing that the effects of USP6NL depend on its GAP function (Figure 2D-E).

Phosphorylation of AKT by PDK1 on Thr308 and by mTORC2 on Ser473 results in full AKT activation (33). USP6NL silencing similarly impaired both phosphorylation events, and the phosphorylation of the AKT substrate GSK3 $\beta$, in the overexpressing BC lines but not in MCF10A cells (Supplementary Figure S3A).

Finally, expression of the myristoylated, constitutive active, PI3K mutant (PI3KCA) (34) recovered AKT activation in USP6NL-silenced cells (Figure 2F). PI3KCA also rescued the defect caused by USP6NL depletion on cell proliferation (Figure 2G) suggesting that down modulation of the PI3K/AKT axis might account for this latter effect in the USP6NL-silenced BC cells.

\section{USP6NL depletion accelerates EGFR degradation downregulating AKT}

Since the GAP function of USP6NL inhibits EGFR endocytosis $(17,19)$, and controls AKT activation (Figure 2D), we tested whether reduced phosphorylation of AKT in 
USP6NL-silenced cells resulted from increased internalization and degradation of the EGFR. Depletion of USP6NL in the BC-overexpressing cell lines, but not in MCF10A or HMEC cells, accelerated EGFR degradation dampening both EGFR and AKT phosphorylation both at steady-state (Figure 3A) and upon EGF stimulation (Supplementary Figure S3B). Measurements of EGFR endocytosis confirmed that functional ablation of USP6NL in HCC70 cells increased the amount of internalized EGFR (Supplementary Figure S3C). Conversely, another RTK, the MET receptor, was largely unaffected by USP6NL silencing, suggesting some degree of specificity of USP6NL, within the limits of the tested conditions. In particular, total MET levels (Figure 3A) and receptor internalization (Supplementary Figure S3C) were not appreciably affected. Some, not significant, increase was detected in the levels of phospho-MET, not correlating with AKT phosphorylation or USP6NL levels. Together these results are compatible with the notion that overexpression of USP6NL delays EGFR endocytosis and degradation, thereby chronically activating AKT.

A likely downstream candidate for the effects of USP6NL in the described phenotypes is RAB5. USP6NL exerts GAP activity towards several Rabs (17-20), among these, RAB5 has been directly implicated in endocytic pathways and specifically in EGFR endocytosis $(35,36)$. We tested, therefore, whether overexpression of RAB5 could phenocopy the ablation of USP6NL. This was indeed the case, as shown by reduced EGFR levels, and AKT phosphorylation (Figure 3B) implicating RAB5 as a probable downstream effector of USP6NL in the control of the analyzed phenotypes.

USP6NL overexpression fuels aerobic glycolysis in BC cells by stabilizing the glucose transporter GLUT1 in an AKT-dependent manner 
AKT controls cell proliferation, survival and metabolism (33). Thus, we looked at the ATP cell content and at the occurrence of cell death in the USP6NL-silenced cell lines. In these experiments, we introduced also a lung carcinoma cell line, H1299, which has little amount of USP6NL, to test the effects in a transformed background bearing low USP6NL levels (Supplementary Figure S4A-B). Depletion of USP6NL reduced the ATP cellular content in HCC70 and HCC1187, but not in USP6NL-low cells (Figure 4A) without causing significant differences in cell death, as measured by DNA accessibility (Figure 4B) suggesting a role for USP6NL in the metabolic control of cancer cell growth.

One of the best-characterized metabolic outcomes of the upregulation of AKT in cancer is increased aerobic glycolysis (37). In USP6NL-depleted BC lines, but not in USP6NL-Iow MCF10A and H1299 cells, we found a 80\% decrease in the rate of glucose uptake (Figure 5A). Similarly, lactate production was significantly reduced (Figure 5B). Finally, only the USP6NL-overexpressing BC cell lines were sensitive to glucose deprivation (Figure 5C). Notably, USP6NL depletion abrogated this phenotype (Figure 5C) indicating that the elevated glycolytic metabolism of these cells depends on the high USP6NL levels.

AKT stimulates the glycolytic capacity of cancer cells by increasing expression and targeting of GLUT1 to the PM, and by regulating key glycolytic enzymes (1416,37). In particular, AKT stabilizes GLUT1 at the PM by inhibiting its endocytosis $(12,13)$. In agreement, we found that USP6NL depletion decreased the amount of GLUT1 in the overexpressing BC cell lines, but not in the USP6NL-low cells where, rather, an increment in the glucose transporter was observed (Figure 6A). Reexpression of USP6NL, but not of R150, in the silenced HCC70 cells readily recovered GLUT1 levels showing that the latter depend on the GAP activity, hence - 
most likely - on the endocytic/trafficking function of USP6NL (Figure 6B). In further support, treatment with the lysosomal blocking agent bafilomycin, but not with the proteasomal inhibitor MG132, rescued the levels of GLUT1 in USP6NL-silenced cells indicating that USP6NL stabilizes GLUT1 by inhibiting its trafficking-mediated degradation (Figure 6C). Finally, and in agreement with a GAP-dependent role for USP6NL in GLUT1 endocytosis and degradation, also overexpression of RAB5 reduced GLUT1 levels (Figure 6D).

We analyzed whether the effect of USP6NL on GLUT1 stability are mediated by AKT. The expression of PI3KCA in the USP6NL-silenced HCC70 cells, along with the rescue of AKT phosphorylation (Figure 2F), also recovered GLUT1 expression (Figure 6E). In addition, treatment with the AKT inhibitor MK2206 resulted in GLUT1 degradation, which was rescued by concomitant addition of bafilomycin, exclusively in the USP6NL-overexpressing BC cells (Figure 6F). Induction of GLUT1 degradation in the HCC1187 cells required high inhibitor concentration. However, MK2206 did not cause GLUT1 degradation in the USP6NL-low cells, even at the highest concentration (Figure 6F). Altogether these data argue that increased USP6NL levels stabilize GLUT1 in an AKT dependent-manner.

Next, we tried to obtain evidence linking the stabilization of GLUT1 in USP6NLoverexpressing cells to AKT-dependent inhibition of endocytosis of the transporter. We analyzed the dynamics of GLUT1 transport upon EGF stimulation in HMEC cells overexpressing USP6NL (USP6NL high) or its GAP mutant $\left(\mathrm{R} 150^{\text {high }}\right)$ (Figure 7A and Supplementary Figure S5A). In control HMEC, in the absence of stimulation (SF), GLUT1 mainly localized in intracellular compartments, partially overlapping with the early endosomal marker EEA1. Stimulation with EGF for 5 min caused significant redistribution to the $\mathrm{PM}$ and reduced co-localization with EEA1. When EGF was 
removed to interrupt stimulation, GLUT1 re-localized to early endosomes. Similar dynamics were observed in HMEC-R150 high cells. Conversely, in HMEC-USP6NL ${ }^{\text {high }}$, GLUT1 was predominantly at the PM under all conditions, including in serum starved cells, suggesting that increased USP6NL levels sustain basal AKT activation that, in turn, maintains GLUT1 at the cell surface. In support, we observed basal activation of AKT in serum starved USP6NL ${ }^{\text {high }}$ cells (Supplementary Figure S6A-B). Moreover, consistent with data showing that AKT blockade prevents localization of glucose transporters to the PM (38), pre-treatment with MK2206 prompted GLUT1 removal from the surface in control and R150 high cells stimulated with EGF as well as in the serum-starved USP6NL ${ }^{\text {high }}$ cells (Figure 7B and Supplementary Figure S5A). The ability of USP6NL to interfere with GLUT1 endocytic dynamics was also confirmed by two additional approaches exploiting either a GLUT1 construct carrying an exofacial myc tag or surface biotinylation experiments (Supplementary Figure S5BC). Finally, similarly to USP6NL depletion, inhibition of AKT by MK2206 treatment impaired proliferation in the BC cell lines overexpressing USP6NL (Supplementary Figure S7).

In conclusion, our findings argue that high USP6NL levels stabilize GLUT1 in an AKT-dependent manner fostering aerobic glycolysis, and determine sensitivity to glucose deprivation in $\mathrm{BC}$ cells. 


\section{DISCUSSION}

Our study reveals a previously unrecognized crosstalk between endocytosis and metabolic reprogramming in $\mathrm{BC}$ relying on the overexpression of USP6NL. This event is likely to play a causal role in the natural history of $\mathrm{BC}$, as USP6NL is frequently amplified/overexpressed in this cancer, in particular in basal-like tumors classified as IntClust10, and correlates with worse prognostic outcome. In addition, in vitro, the depletion of USP6NL impairs proliferation exclusively of BC cells overexpressing this protein and not of normal mammary cells. This suggests a degree of addiction of USP6NL-overexpressing BCs, pointing to this alteration as a relevant mechanism of signaling-metabolic rewiring.

Under basal conditions in BC cells that overexpress USP6NL, functional depletion of the latter promotes EGFR and AKT downregulation. We hypothesize that chronic USP6NL overexpression, by delaying EGFR endocytosis (probably by inhibiting the action of RAB5), stabilizes the receptor at the PM facilitating its activation and the ensuing recruitment of PI3K, eventually resulting in sustained AKT phosphorylation. This hypothesis is supported by the observation that ectopic expression of USP6NL in HMEC cells increases AKT phosphorylation even in absence of exogenous EGFR stimulation. Furthermore, USP6NL is mainly localized to the PM (19), a location where the PI3K/AKT signaling axis is generally considered to initiate (39). Finally, AKT phosphorylates the GLUT1 endocytic adaptor TXNIP at the PM, inhibiting the endocytosis of glucose transporters (40). Thus, the concerted action of USP6NL and AKT, acting at the cell surface, might link EGFR signaling to glucose uptake.

Similarly to the EGFR, also GLUT1 degradation is accelerated by USP6NL depletion, and by AKT inhibition through MK2206, only in USP6NL-overexpressing BC cells. Interestingly, MK2206 is an allosteric AKT inhibitor that prevents insulin- 
driven localization of GLUT1 to the PM and glucose uptake (38) but does not cause GLUT1 degradation in normal cells [this study and (38)]. In agreement, only cell lines overexpressing USP6NL are sensitive to AKT inhibition and glucose starvation. Of note, depletion of USP6NL abrogates sensitivity to glucose deprivation, in the same cell lines, further indicating that the high glycolytic capacity of these cells relies on USP6NL.

Based on the sum of our results, it is tempting to speculate that the poor prognostic outcome of BC patients carrying high USP6NL copy number might be related to the ability of USP6NL to promote glycolysis. Indeed, elevation of glycolysis is generally associated with tumor aggressiveness and poor prognosis, and is a distinguishing feature of a subset of basal-like BCs (2,3). Interestingly, in many instances, upregulation of glycolysis in tumors occurs through overexpression/stabilization of GLUT1, an alteration that correlates with aggressiveness (41-45). In this context, our data argue that the amplification/overexpression of USP6NL might be a mechanism of metabolic adaptation/fitness for some BCs. If so, USP6NL overexpression might represent a point of metabolic vulnerability for a subset of aggressive basal-like breast tumors. 


\section{ACKNOWLEDGMENTS}

We thank Miriam Martini and Emilio Hirsch for helpful discussion and for reagents, Carlos Sebastian for suggestions and for critically reading the manuscript. Davide Disalvatore for bioinformatics support. Work in the authors' lab is supported by grants from the Associazione Italiana per la Ricerca sul Cancro (AIRC Investigator Grant, project 15180 to L.L. and 14404, MCO 10.000 and extension to PPDF, 5xmille MCO Extension to AS), Fondo Ricerca Locale 2017 (University of Turin) to LL, MIUR (the Italian Ministry of University and Scientific Research) to PPDF, the Monzino Foundation to PPDF, FPRC 5xmille Ministero Salute 2011, 2014 to AS and 2015 to LL, and Cancer UK to A.B., O.M.R. and C.C. 


\section{REFERENCES}

1. Lehmann BD, Bauer JA, Chen X, Sanders ME, Chakravarthy AB, Shyr Y, et al. Identification of human triple-negative breast cancer subtypes and preclinical models for selection of targeted therapies. J Clin Invest 2011;121:2750-67

2. Palaskas N, Larson SM, Schultz N, Komisopoulou E, Wong J, Rohle D, et al. 18Ffluorodeoxy-glucose positron emission tomography marks MYC-overexpressing human basal-like breast cancers. Cancer Res 2011;71:5164-74

3. McCleland ML, Adler AS, Shang Y, Hunsaker T, Truong T, Peterson D, et al. An integrated genomic screen identifies LDHB as an essential gene for triple-negative breast cancer. Cancer Res 2012;72:5812-23

4. Barron CC, Bilan PJ, Tsakiridis T, Tsiani E. Facilitative glucose transporters: Implications for cancer detection, prognosis and treatment. Metabolism 2016;65:12439

5. Eyster CA, Higginson JD, Huebner R, Porat-Shliom N, Weigert R, Wu WW, et al. Discovery of new cargo proteins that enter cells through clathrin-independent endocytosis. Traffic 2009; $10: 590-9$

6. Wu N, Zheng B, Shaywitz A, Dagon Y, Tower C, Bellinger G, et al. AMPKdependent degradation of TXNIP upon energy stress leads to enhanced glucose uptake via GLUT1. Mol Cell 2013;49:1167-75

7. Kvainickas A, Orgaz AJ, Nägele H, Diedrich B, Heesom KJ, Dengjel J, et al. Retromer- and WASH-dependent sorting of nutrient transporters requires a multivalent interaction network with ANKRD50. J Cell Sci 2017;130:382-95

8. Steinberg F, Gallon M, Winfield M, Thomas EC, Bell AJ, Heesom KJ, et al. A global analysis of SNX27-retromer assembly and cargo specificity reveals a function in glucose and metal ion transport. Nat Cell Biol 2013;15:461-71

9. Roy S, Leidal AM, Ye J, Ronen SM, Debnath J. Autophagy-Dependent Shuttling of TBC1D5 Controls Plasma Membrane Translocation of GLUT1 and Glucose Uptake. Mol Cell 2017;67:84-95.e5

10. Wieman HL, Horn SR, Jacobs SR, Altman BJ, Kornbluth S, Rathmell JC. An essential role for the Glut1 PDZ-binding motif in growth factor regulation of Glut1 degradation and trafficking. Biochem J 2009;418:345-67

11. Antonescu CN, McGraw TE, Klip A. Reciprocal regulation of endocytosis and metabolism. Cold Spring Harb Perspect Biol 2014;6:a016964

12. Wieman HL, Wofford JA, Rathmell JC. Cytokine stimulation promotes glucose uptake via phosphatidylinositol-3 kinase/Akt regulation of Glut1 activity and trafficking. Mol Biol Cell 2007;18:1437-46

13. Wofford JA, Wieman HL, Jacobs SR, Zhao Y, Rathmell JC. IL-7 promotes Glut1 trafficking and glucose uptake via STAT5-mediated activation of Akt to support Tcell survival. Blood 2008;111:2101-11

14. Cairns RA, Harris IS, Mak TW. Regulation of cancer cell metabolism. Nat Rev Cancer 2011;11:85-95

15. Hong SY, Yu FX, Luo Y, Hagen T. Oncogenic activation of the PI3K/Akt pathway promotes cellular glucose uptake by downregulating the expression of thioredoxininteracting protein. Cell Signal 2016;28:377-83

16. Makinoshima H, Takita M, Saruwatari K, Umemura S, Obata Y, Ishii G, et al. Signaling through the Phosphatidylinositol 3-Kinase (PI3K)/Mammalian Target of Rapamycin (mTOR) Axis Is Responsible for Aerobic Glycolysis mediated by Glucose Transporter in Epidermal Growth Factor Receptor (EGFR)-mutated Lung Adenocarcinoma. J Biol Chem 2015;290:17495-504 
17. Lanzetti L, Rybin V, Malabarba MG, Christoforidis S, Scita G, Zerial M, et al. The Eps8 protein coordinates EGF receptor signalling through Rac and trafficking through Rab5. Nature 2000;408:374-7.

18. Lanzetti L, Palamidessi A, Areces L, Scita G, Di Fiore PP. Rab5 is a signalling GTPase involved in actin remodelling by receptor tyrosine kinases. Nature 2004;429:309-14

19. Palamidessi A, Frittoli E, Ducano N, Offenhauser N, Sigismund S, Kajiho H, et al. The GTPase-activating protein $\mathrm{RN}$-tre controls focal adhesion turnover and cell migration. Curr Biol 2013;23:2355-64

20. Haas AK, Yoshimura S, Stephens DJ, Preisinger C, Fuchs E, Barr FA. Analysis of GTPase-activating proteins: Rab1 and Rab43 are key Rabs required to maintain a functional Golgi complex in human cells. J Cell Sci 2007;120:2997-3010

21. Malinverno C, Corallino S, Giavazzi F, Bergert M, Li Q, Leoni M, et al. Endocytic reawakening of motility in jammed epithelia. Nat Mater 2017

22. Zhang C, Liu J, Liang Y, Wu R, Zhao Y, Hong X, et al. Tumour-associated mutant p53 drives the Warburg effect. Nat Commun 2013;4:2935

23. Curtis C, Shah SP, Chin SF, Turashvili G, Rueda OM, Dunning MJ, et al. The genomic and transcriptomic architecture of 2,000 breast tumours reveals novel subgroups. Nature 2012;486:346-52

24. Ritchie ME, Phipson B, Wu D, Hu Y, Law CW, Shi W, et al. limma powers differential expression analyses for RNA-sequencing and microarray studies. Nucleic Acids Res 2015;43:e47

25. Pupo E, Ducano N, Lupo B, Vigna E, Avanzato D, Perera T, et al. Rebound effects caused by withdrawal of MET kinase inhibitor are quenched by a MET therapeutic antibody. Cancer Res 2016

26. Serio G, Margaria V, Jensen S, Oldani A, Bartek J, Bussolino F, et al. Small GTPase Rab5 participates in chromosome congression and regulates localization of the centromere-associated protein CENP-F to kinetochores. Proc Natl Acad Sci U S A 2011;108:17337-42

27. Gao J, Aksoy BA, Dogrusoz U, Dresdner G, Gross B, Sumer SO, et al. Integrative analysis of complex cancer genomics and clinical profiles using the cBioPortal. Sci Signal 2013;6:pl1

28. Cerami E, Gao J, Dogrusoz U, Gross BE, Sumer SO, Aksoy BA, et al. The cBio cancer genomics portal: an open platform for exploring multidimensional cancer genomics data. Cancer Discov 2012;2:401-4

29. Pereira B, Chin SF, Rueda OM, Vollan HK, Provenzano E, Bardwell HA, et al. The somatic mutation profiles of 2,433 breast cancers refines their genomic and transcriptomic landscapes. Nat Commun 2016;7:11479

30. Ciriello G, Gatza ML, Beck AH, Wilkerson MD, Rhie SK, Pastore A, et al. Comprehensive Molecular Portraits of Invasive Lobular Breast Cancer. Cell 2015; $163: 506-19$

31. Dawson SJ, Rueda OM, Aparicio S, Caldas C. A new genome-driven integrated classification of breast cancer and its implications. EMBO J 2013;32:617-28

32. Lanzetti L, Di Fiore PP. Behind the Scenes: Endo/Exocytosis in the Acquisition of Metastatic Traits. Cancer Res 2017

33. Manning BD, Toker A. AKT/PKB Signaling: Navigating the Network. Cell 2017;169:381-405

34. Zhao JJ, Liu Z, Wang L, Shin E, Loda MF, Roberts TM. The oncogenic properties of mutant p110alpha and p110beta phosphatidylinositol 3-kinases in human mammary epithelial cells. Proc Natl Acad Sci U S A 2005; $102: 18443-8$ 
35. Papini E, Satin B, Bucci C, de Bernard M, Telford JL, Manetti R, et al. The small GTP binding protein rab7 is essential for cellular vacuolation induced by Helicobacter pylori cytotoxin. Embo J 1997; 16:15-24

36. Barbieri MA, Roberts RL, Gumusboga A, Highfield H, Alvarez-Dominguez C, Wells A, et al. Epidermal growth factor and membrane trafficking. EGF receptor activation of endocytosis requires Rab5a. J Cell Biol 2000;151:539-50

37. Elstrom RL, Bauer DE, Buzzai M, Karnauskas R, Harris MH, Plas DR, et al. Akt stimulates aerobic glycolysis in cancer cells. Cancer Res 2004;64:3892-9

38. Beg M, Abdullah N, Thowfeik FS, Altorki NK, McGraw TE. Distinct Akt phosphorylation states are required for insulin regulated Glut4 and Glut1-mediated glucose uptake. Elife 2017;6

39. Sorkin A, von Zastrow M. Endocytosis and signalling: intertwining molecular networks. Nat Rev Mol Cell Biol 2009;10:609-22

40. Waldhart AN, Dykstra H, Peck AS, Boguslawski EA, Madaj ZB, Wen J, et al. Phosphorylation of TXNIP by AKT Mediates Acute Influx of Glucose in Response to Insulin. Cell Rep 2017;19:2005-13

41. Gatenby RA, Gillies RJ. Why do cancers have high aerobic glycolysis? Nat Rev Cancer 2004;4:891-9

42. Yang J, Wen J, Tian T, Lu Z, Wang Y, Wang Z, et al. GLUT-1 overexpression as an unfavorable prognostic biomarker in patients with colorectal cancer. Oncotarget 2016

43. Kunkel M, Reichert TE, Benz P, Lehr HA, Jeong JH, Wieand S, et al. Overexpression of Glut-1 and increased glucose metabolism in tumors are associated with a poor prognosis in patients with oral squamous cell carcinoma. Cancer 2003;97:1015-24

44. Maki Y, Soh J, Ichimura K, Shien K, Furukawa M, Muraoka T, et al. Impact of GLUT1 and Ki-67 expression on early- stage lung adenocarcinoma diagnosed according to a new international multidisciplinary classification. Oncol Rep 2013;29:133-40

45. Lastraioli E, Bencini L, Bianchini E, Romoli MR, Crociani O, Giommoni E, et al. hERG1 Channels and Glut-1 as Independent Prognostic Indicators of Worse Outcome in Stage I and II Colorectal Cancer: A Pilot Study. Transl Oncol 2012;5:105-12 


\section{FIGURE LEGENDS}

\section{Figure 1. USP6NL overexpression in BC}

A) Venn diagrams showing the number of cases harboring increased USP6NL copy number (AMP, red) and mRNA overexpression (UP-REG, black) in the METABRIC (left) and TCGA (right) datasets. $p$, p-value of the association between the indicated variables by Fisher's exact test. B) Correlation between USP6NL copy number and mRNA levels in the METABRIC samples ( $\mathrm{N}=1974)$. DEL=copy number deletion, NEUT=copy number neutral, GAIN= copy number gain, AMP=copy number amplification; association between AMP and mRNA expression $p<0.0001$. C) USP6NL mRNA levels in the Pam50 molecular subtypes in the METABRIC dataset. $\mathrm{N}=$ normal breast tissue, Norm $=$ Normal-like, $\operatorname{Lum} A=$ luminal $A$, LumB $=$ luminal $B$, Her2= Her2 amplified, Basal= basal-like; correlation between overexpression and Basal tumors $p<0.0001$. D) Kaplan-Meir plots showing the disease-specific survival in patients with (gain) or without (neutral/loss) copy number gain in the METABRIC dataset. HR, hazard ratio univariate gain vs. neutral/loss. $\mathrm{HR}^{*}$, hazard ratio multivariable gain vs. neutral/loss. E) Representative images of USP6NL protein expression by IHC in TMA cores and their relative score. F) Distribution of USP6NL protein expression according to the IHC score in normal breast tissue (99 samples) and BCs (1608 samples).

Figure 2. USP6NL depletion impairs AKT phosphorylation and cell proliferation in USP6NL-overexpressing BC cells

A) Lysates from the indicated cell lines were silenced with control oligos (siCTR) or USP6NL oligo (siUSP6NL) and immunoblotted as indicated. B) Cell counts, after 2 days of growth of HCC70, HCC1187, MCF10A and HMEC cell lines silenced as in 
the legend. Normalization was performed vs. control in each cell line $(\operatorname{siCTR}=1)$. Means \pm s.e.m. from at least 4 independent experiments, each including at least 4 technical replicates. C) Heat maps of phosphoprotein levels in the indicated cell lines silenced or not for USP6NL. The color scale bar represents log2 phosphoprotein levels, relative to control (three independent experiments performed in duplicates). Phospho-AKT $p<0.0001$ in HCC70 and $<0.002$ in HCC1187 cells; pJNK $p<0.0002$ in HCC1187 and <0.002 in MCF10A cells. D) Stable doxy-inducible HCC70 cell populations carrying silencing-resistant USP6NL or the R150 mutant were silenced as indicated on top (siCTR or siUSP6NL), and treated (+) or not (-) with doxycycline for 48 hours to re-express USP6NL or R150. Lysates were immunoblotted as indicated. Densitometric analysis of pAKT/AKT tot is reported underneath. Means \pm s.e.m. $n=4$. Membranes here and in the following Figures, unless otherwise indicated, were first immunoblotted with anti-phospho antibodies, stripped and reprobed with anti-total antibodies. E) Cell counts in the inducible HCC70 populations silenced and treated as in $D$ and indicated in the legend. Means \pm s.e.m. $n=6$; at least 3 technical replicates. F) Lysates from HCC70 cells stably expressing the myristoylated PI3KCA mutant (PI3KCA), or empty vector (mock), were silenced as indicated on top, and immunoblotted as indicated. Densitometric analysis of pAKT/AKT tot is reported underneath. Means \pm s.e.m. $n=6$. G) Cell counts for the cellular populations transfected and silenced as in F. Means \pm s.e.m. $n=5 ; 4$ technical replicates.

Figure 3. USP6NL depletion accelerates EGFR down regulation in the overexpressing BC cells 
A) Left. Lysates from the indicated cell lines were silenced as on top, followed by immunoblotting as shown. Right. Densitometric analyses. Means \pm s.e.m. of at least 3 experiments. B) Stable doxy-inducible MCF10A cell populations carrying the empty vector (CTR) or RAB5 (RAB5) were treated with doxycycline for 48 hours to overexpress RAB5. Total lysates were immunoblotted as on the right. Densitometric analysis is reported on the right. Mean \pm s.e. $m \mathrm{n}=3$.

Figure 4. USP6NL silencing decreases the ATP cell content in the overexpressing BC cells without causing cell death

A-B) The indicated cell lines were silenced as shown in the legend. A) Bioluminescent ATP cell content normalized to siCTR for each cell line $(=1)$. Means \pm s.e.m. from at least 3 independent experiments each point done at least in duplicate. B) Mean fluorescence intensity of the toxicity marker CellTox green \pm s.e.m. $\mathrm{n}=3$ each in technical duplicates.

Figure 5. USP6NL overexpression in BC cells controls their glycolytic metabolism

A) Rate of glucose uptake in the indicated cell lines, silenced as in the legend. Means \pm s.e.m. $n=3$ each in technical duplicates. B) L-Lactate production in HCC70 and $\mathrm{HCC} 1187$ silenced as in the legend. Means \pm s.e.m. $n=3$ each in technical triplicates. C) Sensitivity to glucose deprivation. The indicated cell lines, silenced as in the legend, were grown in complete medium (c.m.) or in medium without glucose (w/o) for 24 hours. Cell counts were normalized vs. their corresponding control in c.m. Means \pm s.e.m. $n=4 ; 4$ technical replicates. 


\section{Figure 6. USP6NL prevents GLUT1-degradation in an AKT-dependent manner}

A) The indicated cell lines were silenced with control (SiCTR) or USP6NL (siUSP6NL) oligos, followed by immunoblotting as indicated. GLUT1 migrates as a broad band at slightly different molecular weights in the various cell lines. B) Stable doxy-inducible HCC70 cells carrying silencing-resistant USP6NL or R150 mutant were silenced as indicated on top and treated (+) or not (-) with doxycycline for 48 hours to re-express USP6NL or R150. Lysates were immunoblotted as shown on the right. C) HCC70 cells, silenced as indicated on top, were treated with DMSO, or bafilomycin (30 nM) or with MG132 (1 $\mu \mathrm{M})$ for 2 hours. Lysates were immunoblotted as on the right. D) Doxy-inducible MCF10A cell populations carrying the empty vector (CTR) or RAB5 (RAB5) were treated with doxycycline for 48 hours to overexpress RAB5 followed by immunoblotting as indicated. E) Lysates from HCC70 cells stably infected with the empty vector (mock) or with the PI3KCA mutant (PI3KCA) and silenced as on top were immunoblotted as on the right. F) Total cellular lysates from the cell lines shown on top treated O/N with DMSO (-) or with MK2206 (+) were immunoblotted as shown. HCC70 and HCC1187 cells were also treated with the combination MK2206 + bafilomycin. In all panels, densitometric analyses of GLUT1 levels are shown underneath as mean \pm s.e.m. In A, B, E, and F, $\mathrm{n}=3$; in $\mathrm{C}$ and $\mathrm{D}, \mathrm{n}=4$.

\section{Figure 7. High USP6NL inhibits AKT-dependent GLUT1 endocytosis}

A) Stable doxy-inducible HMEC cell populations were treated with doxycycline for 48 hours to overexpress HA-tagged USP6NL (USP6NL ${ }^{\text {high }}$ ) or R150 (R150 $\left.{ }^{\text {high }}\right)$. CTR are cells infected with the empty vector. Cells were serum starved O/N (SF) and then treated with $100 \mathrm{ng} / \mathrm{ml}$ of $\mathrm{EGF}$ for $5 \mathrm{~min}$ at $37^{\circ} \mathrm{C}$ (EGF). The ligand was then 
removed by washing and cells were further incubated at $37^{\circ} \mathrm{C}$ for 15 min (EGF removal $15 \mathrm{~min}$ ) before fixation. Confocal images show GLUT1 (green), EEA1 (red), or HA (black and white). In the top rows merged GLUT1 and EEA1 channels are shown and the boxed regions are magnified in the insets underneath (individual channels and merged). B) The indicated cells were treated with $1 \mu \mathrm{M}$ MK2206 for 2 hours and EGF (100 ng/ml) was added to control and R150 high cells 5 min before fixation. Staining as in $A$. Bar in $(A)$ and $(B), 10 \mu \mathrm{m}$. A quantitative analysis of data in this Figure is in Supplementary Figure S5A. 

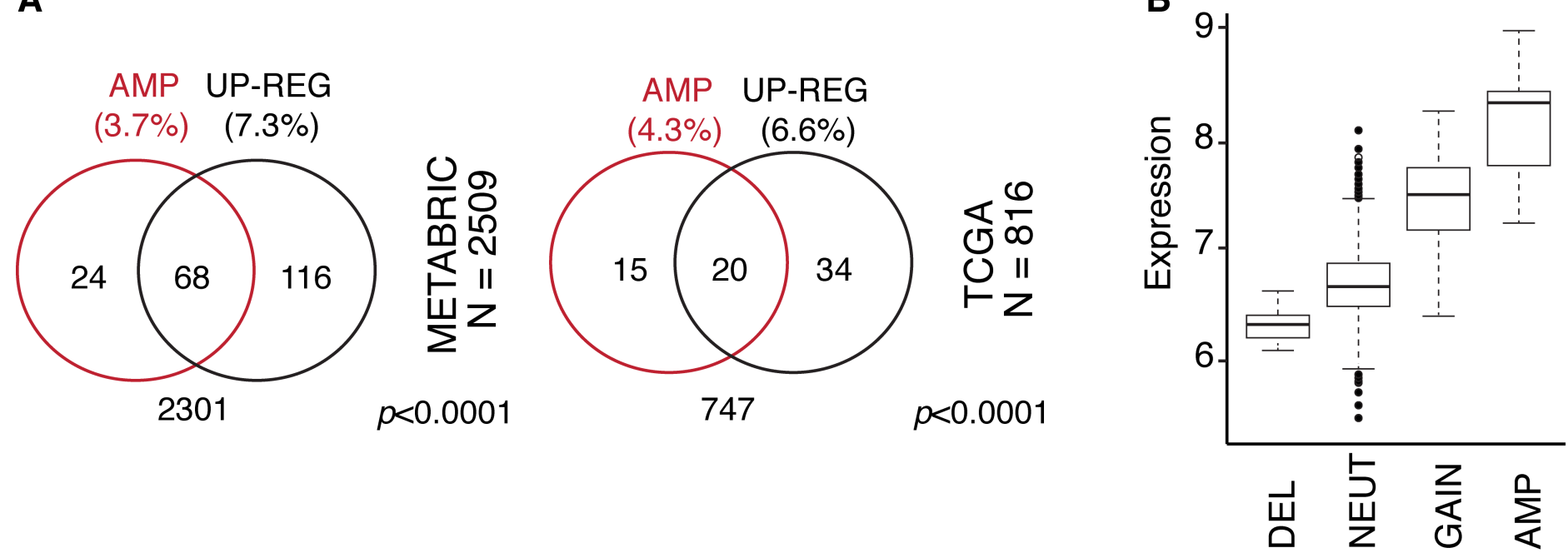

Copy Number

C

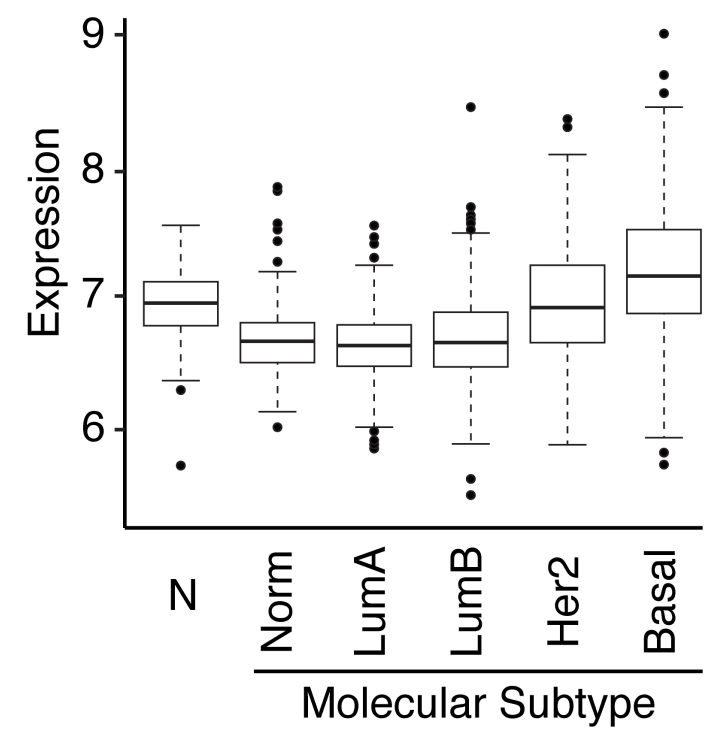

E

IHC staining with USP6NL Assay Biotech C19466 antibody

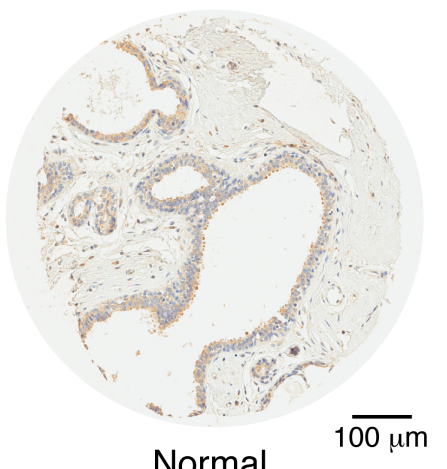

Normal

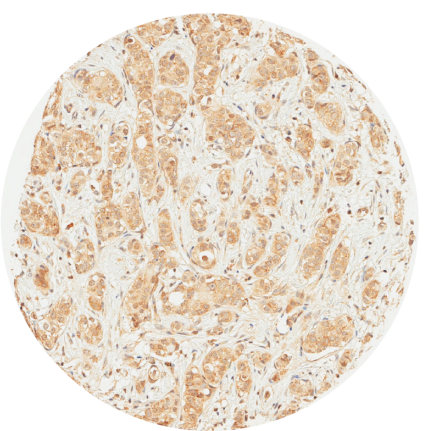

Downloaded

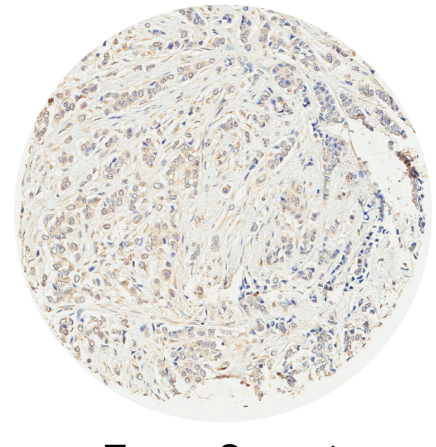

Tumor-Score 1

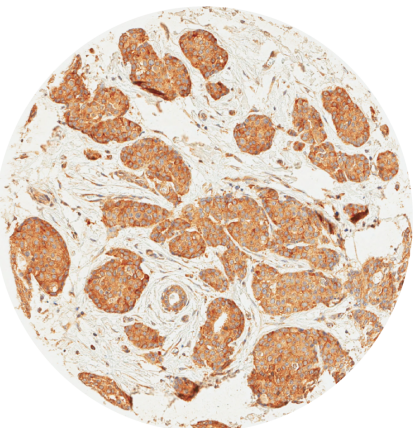

acriournals. org on May 18 Tumor-Score 3 Research.
D

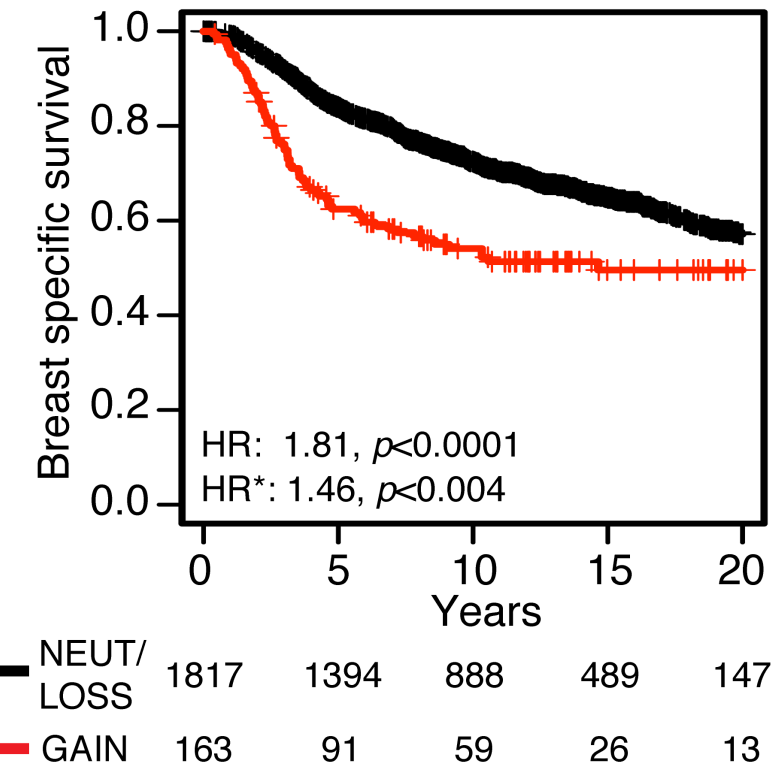

$\mathbf{F}$

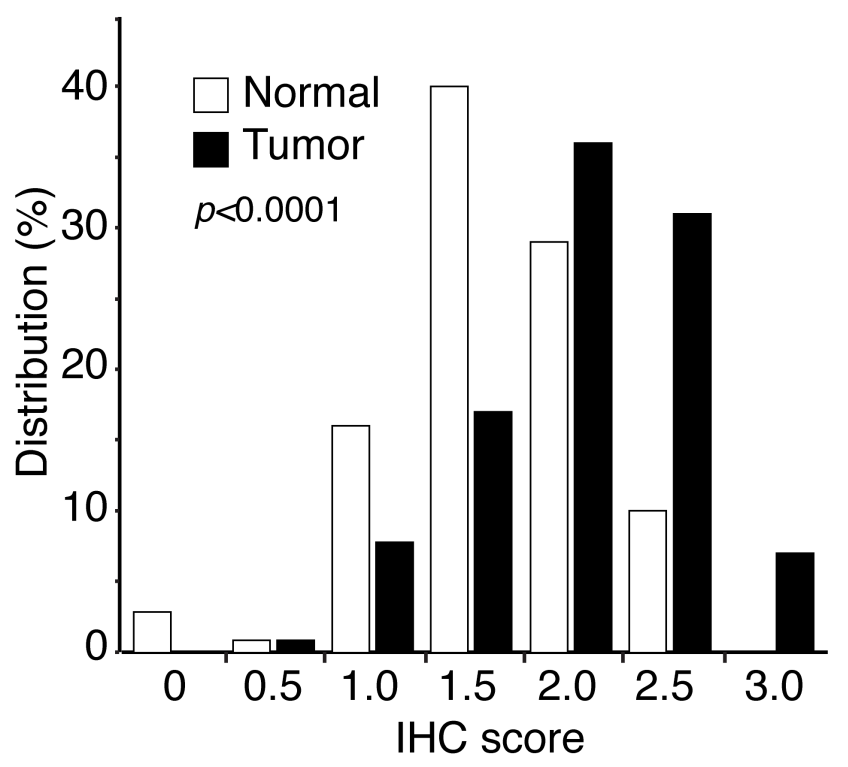


A $\mathrm{HCC} 70$ HCC1187 MCF10A HMEC

siCTR

$\square$ siUSP6NL

$\square$ siUSP6NL+USP6NL

$\square$ siUSP6NL+R150

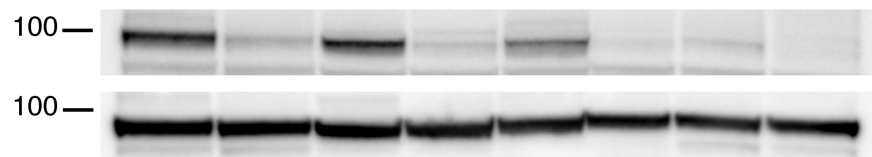

USP6NL

HSP90
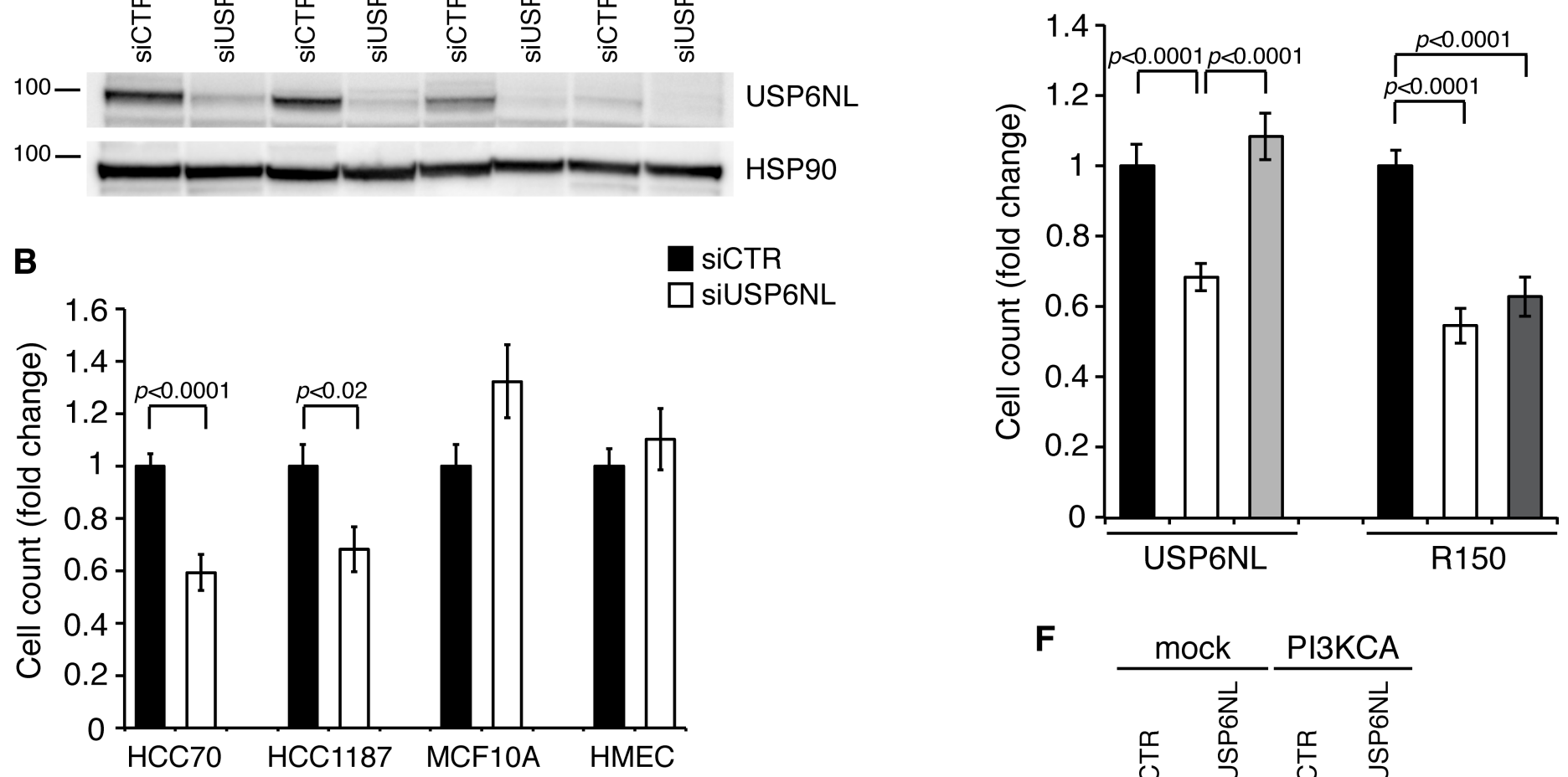

C

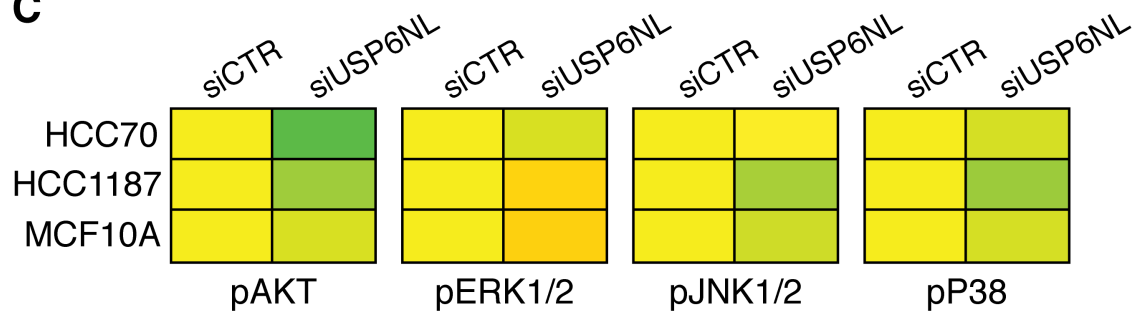

F
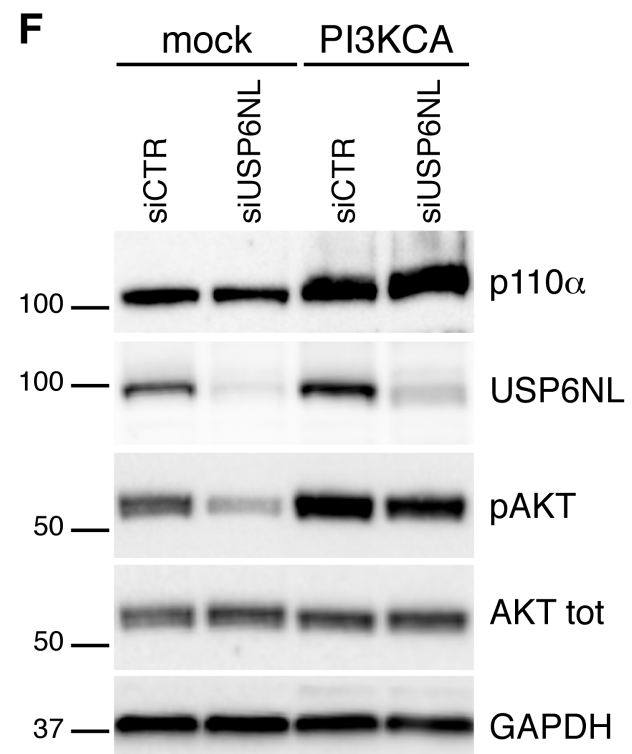

D

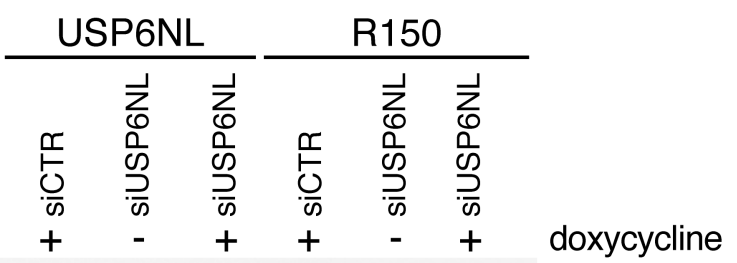

$100-\square-\square$ USP6NL
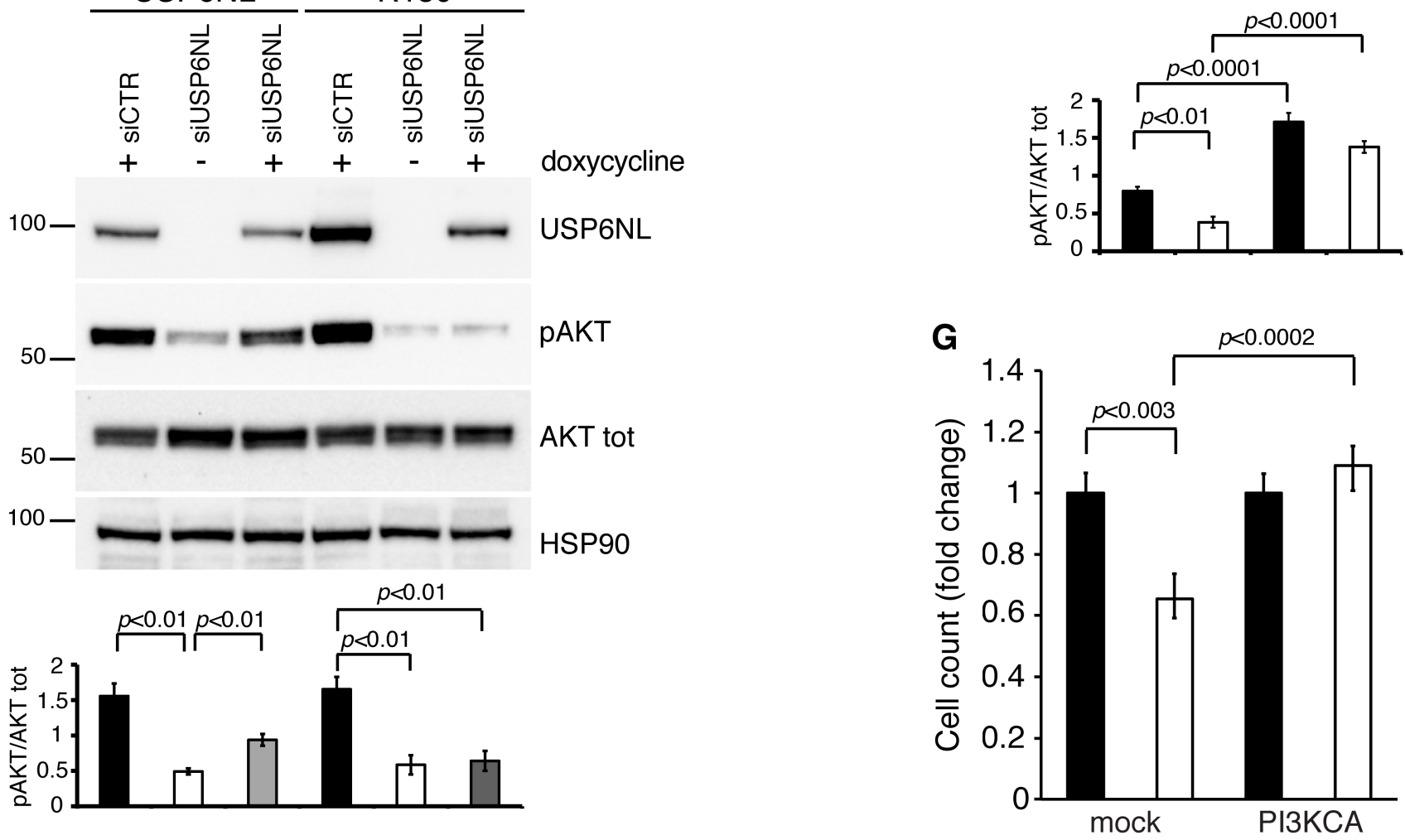

Downloaded from cancerres.aacrjournals.org on May 18, 2018. ( 2018 American Association for Cancer Research.

Figure 2 


\begin{tabular}{|c|c|c|c|c|c|c|c|}
\hline \multicolumn{2}{|c|}{ HMEC } & \multicolumn{2}{|c|}{ MCF10A } & \multicolumn{2}{|c|}{$\mathrm{HCC} 70$} & \multicolumn{2}{|c|}{$\underline{\mathrm{HCC}} 1187$} \\
\hline $\begin{array}{l}\frac{r}{5} \\
\frac{U}{\infty}\end{array}$ & $\begin{array}{l}\sum_{0} \\
0 \\
\frac{1}{\omega} \\
\frac{7}{\omega}\end{array}$ & $\frac{\mathfrak{r}}{\mathfrak{v}}$ & $\begin{array}{l}\frac{1}{2} \\
0 \\
\frac{0}{\infty} \\
\frac{1}{\omega}\end{array}$ & $\begin{array}{l}\frac{\mathfrak{v}}{0} \\
\frac{0}{\omega}\end{array}$ & $\begin{array}{l}\sum \\
0 \\
0 \\
\stackrel{0}{\omega} \\
?\end{array}$ & $\frac{\mathfrak{r}}{\frac{U}{\omega}}$ & $\begin{array}{l}\sum \\
00 \\
0 \\
\frac{0}{\infty} \\
\frac{1}{\omega}\end{array}$ \\
\hline
\end{tabular}

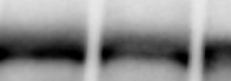

(1)

150

15

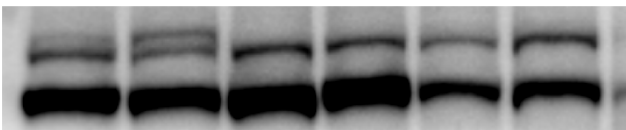

75

50

75

50

100
B

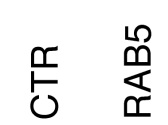

150

$m$ pEGFR

150_ EGFR tot

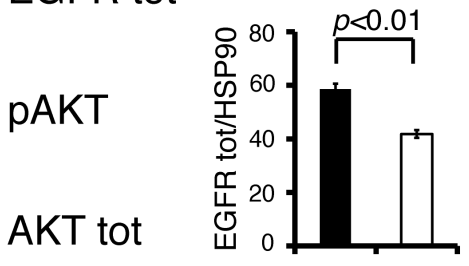

50

25

10

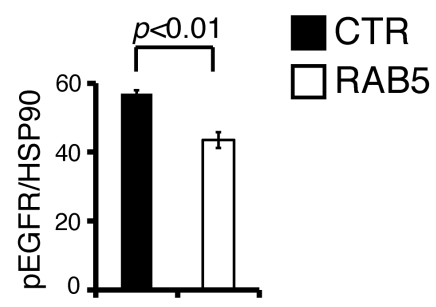

HMEC

MCF10A

HCC70

HCC 1187
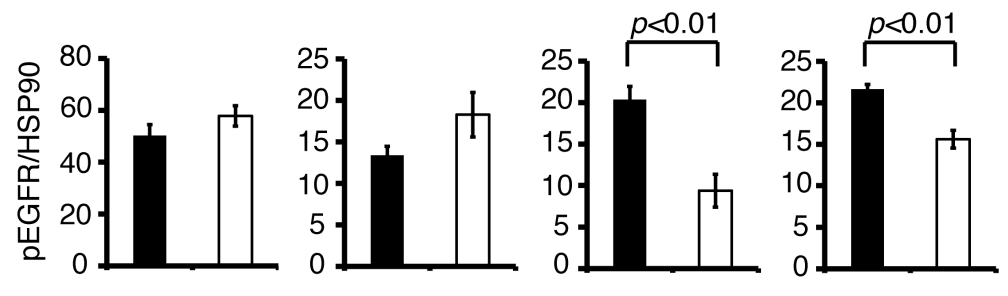

pEGFR
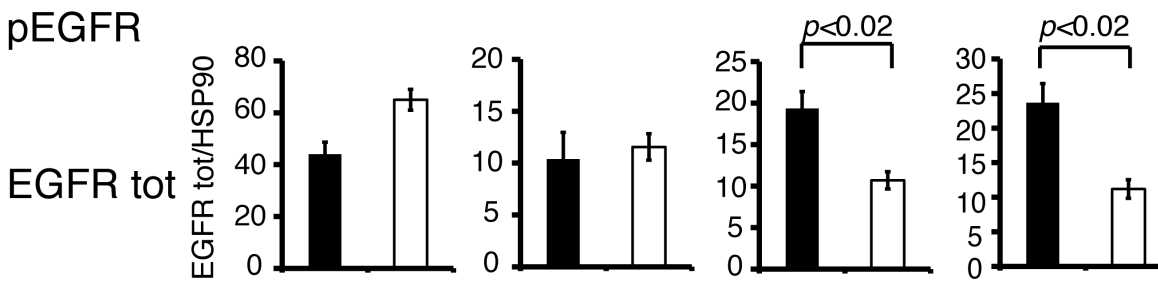

pMET
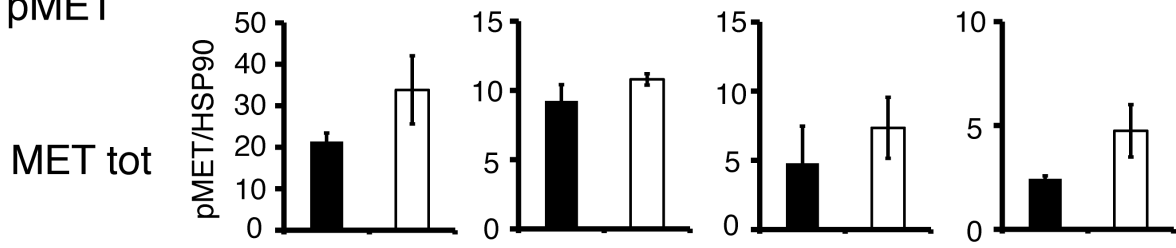

pAKT

AKT tot
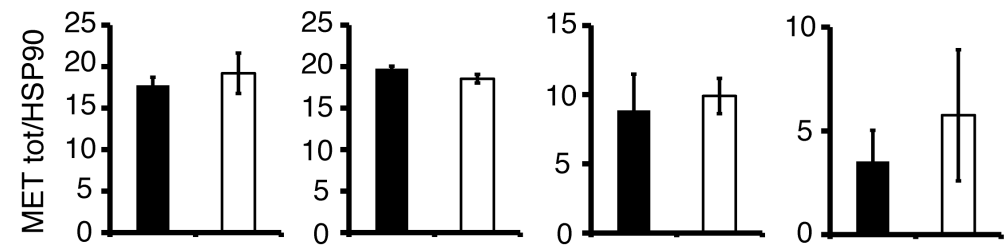

USP6NL
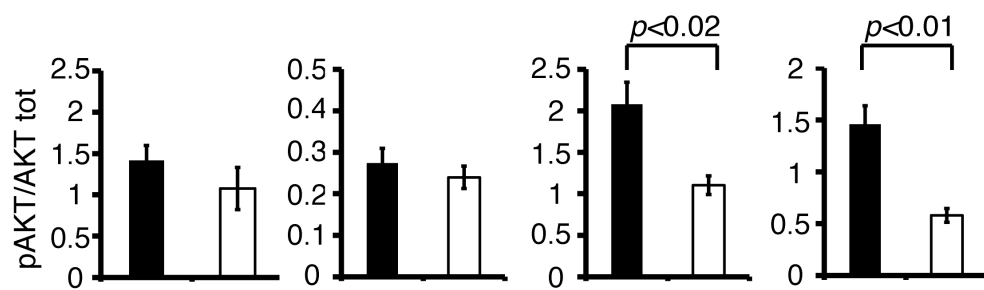

Figure 3 


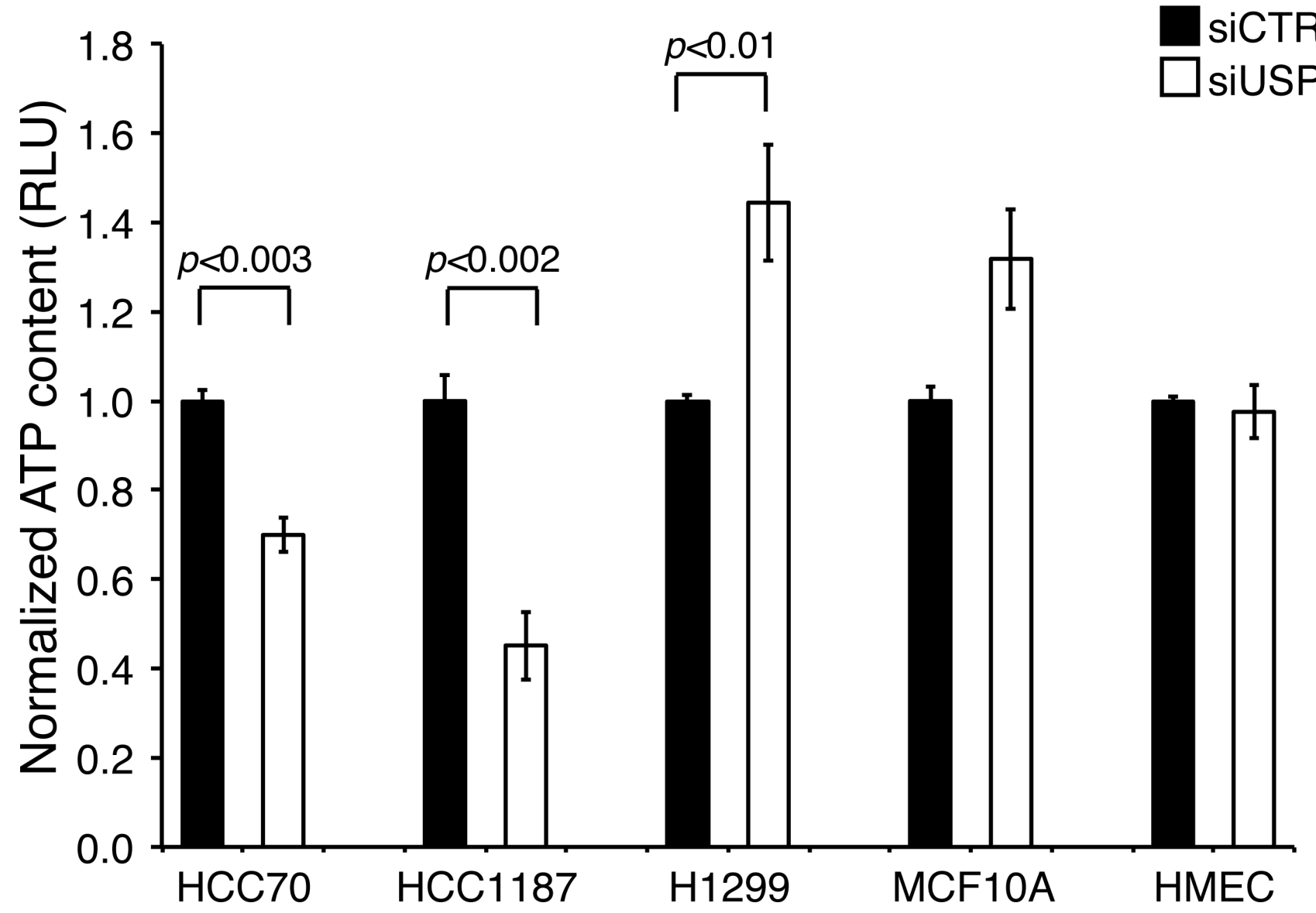

B

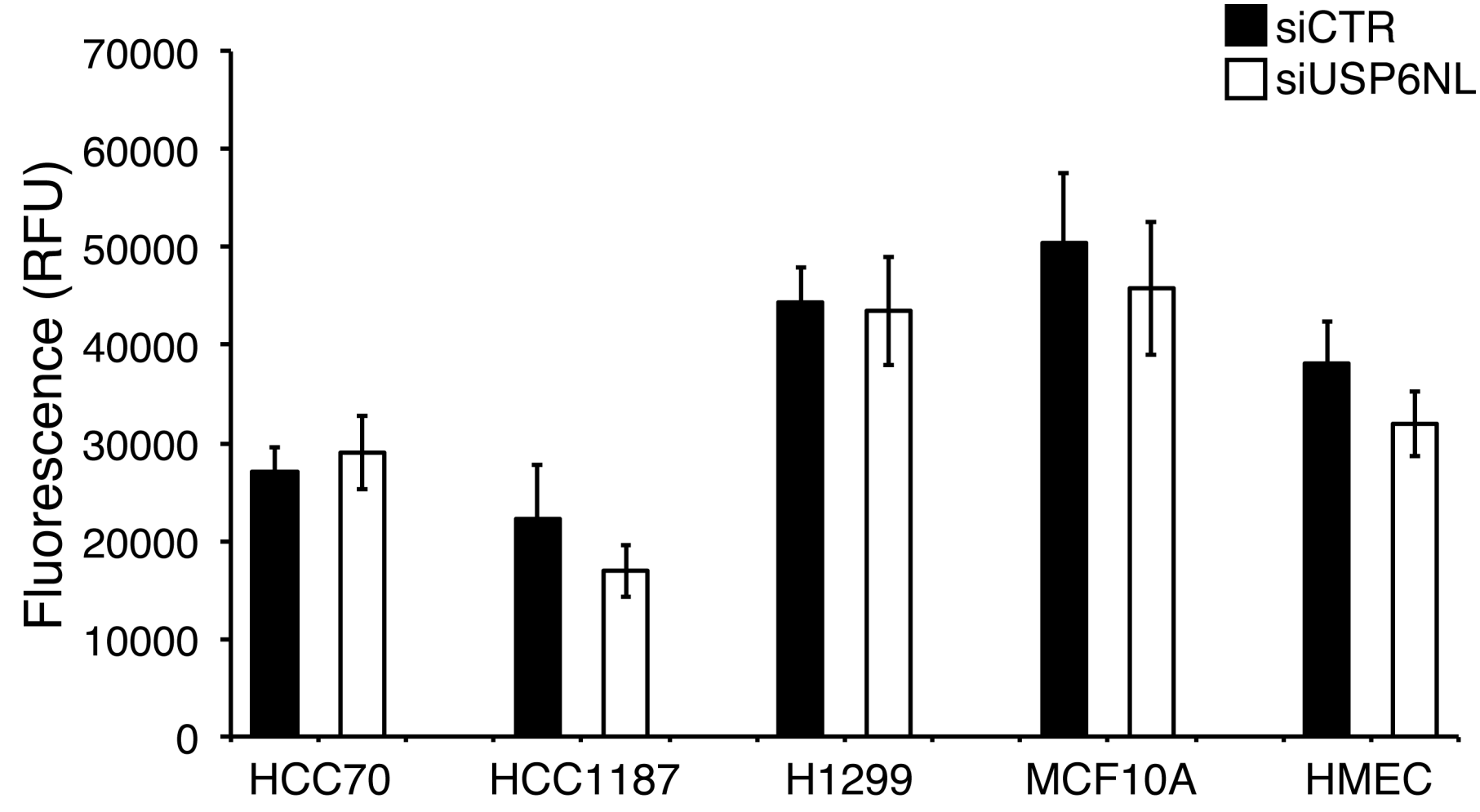


B

SiCTR

$\square$ siUSP6NL

HCC70

HCC1187

MCF10A

H1299
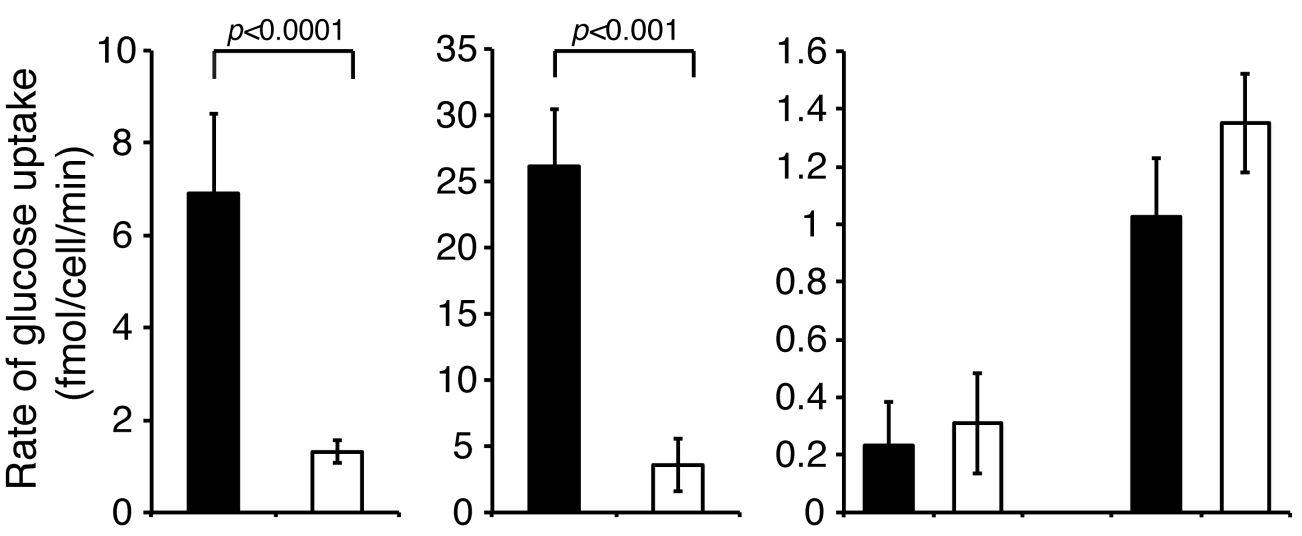

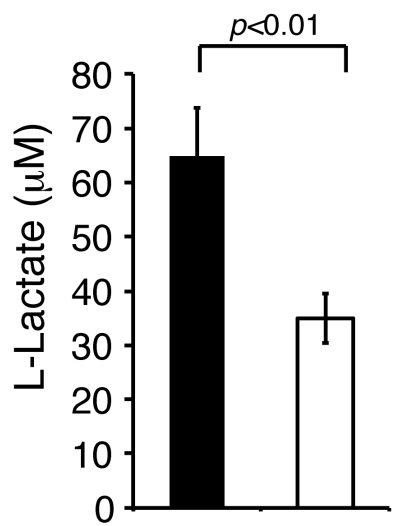

HCC70

SiCTR

$\square$ siUSP6NL

HCC1187

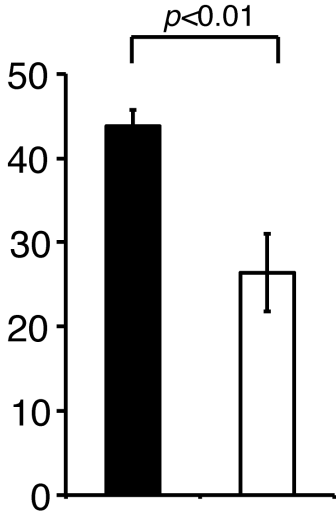

SiCTR

HCC1187

MCF10A

H1299 $\square$ siUSP6NL

$p<0.02$

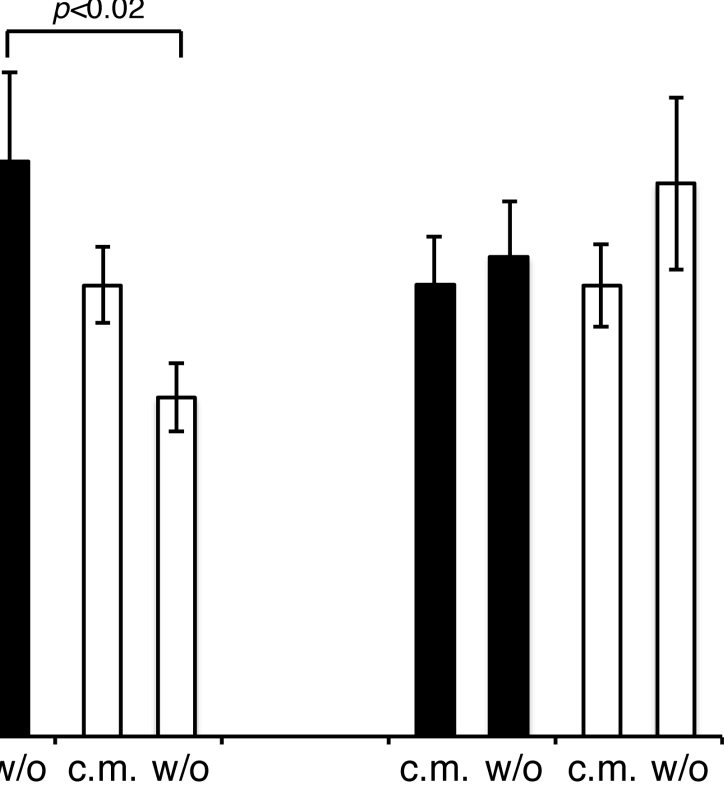

Figure 5 
HCC7O HCC1187 MCF10A HMEC H1209

\begin{tabular}{|c|c|c|c|c|c|}
\hline HCC70 & HCC1187 & MCF10A & HMEC & & 299 \\
\hline 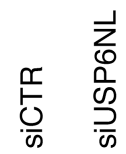 & 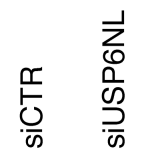 & $\begin{array}{ll} & \sum \\
0 & 0 \\
\frac{x}{0} & 0 \\
\frac{0}{\omega} & \frac{1}{\omega}\end{array}$ & $\begin{array}{l}\sum_{0} \\
0 \\
0 \\
\frac{1}{\omega} \\
\frac{2}{2}\end{array}$ & $\frac{\mathfrak{r}}{\frac{0}{\infty}}$ & $\begin{array}{l}\sum_{0} \\
0 \\
0 \\
\frac{0}{\infty}\end{array}$ \\
\hline
\end{tabular}

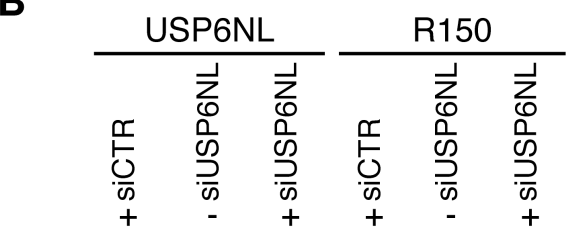

doxycycline

USP6NL

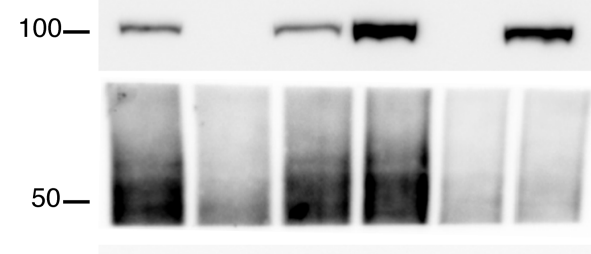

USP6NL
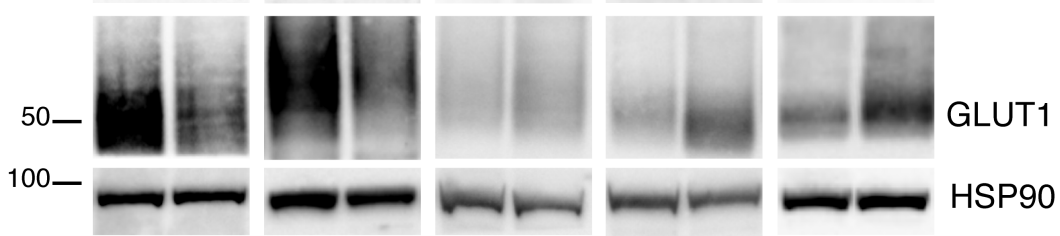

37-

GLUT1

GAPDH
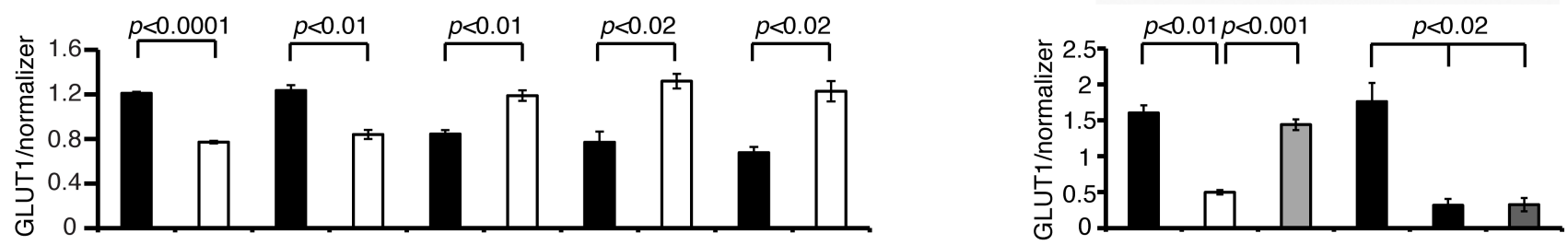

C

D
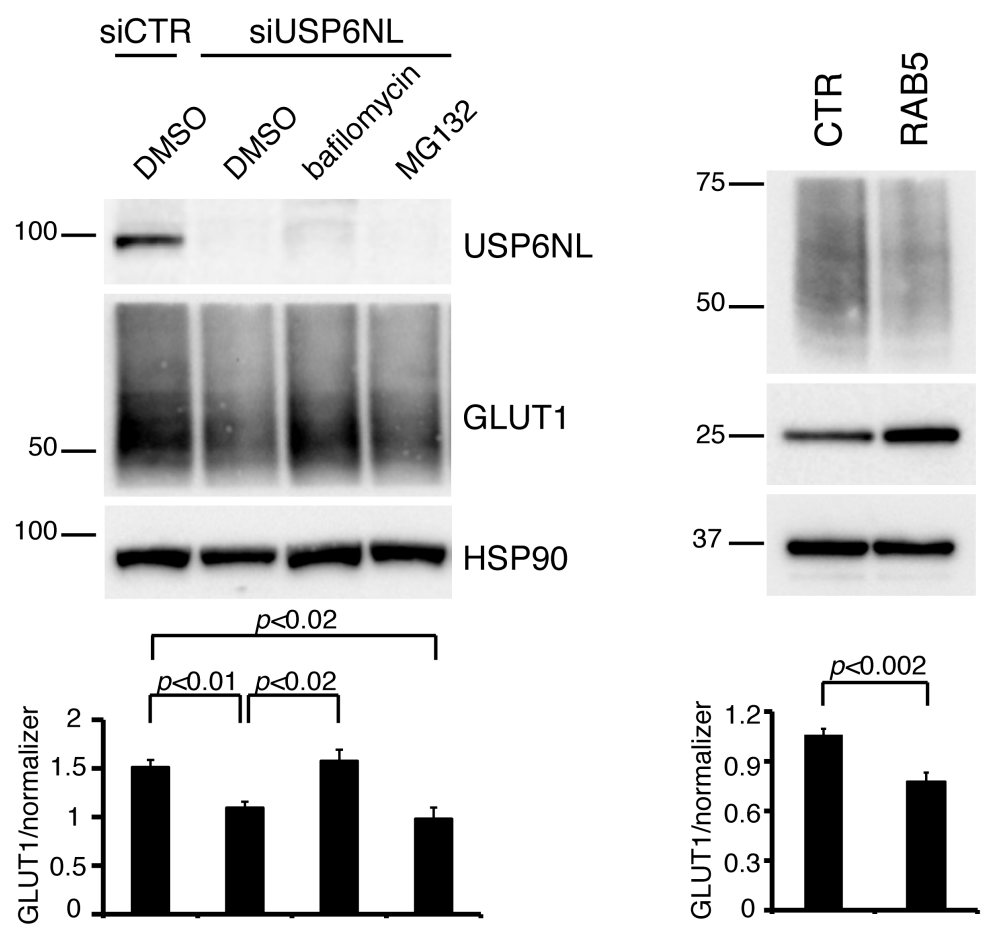

GLUT1

RAB5
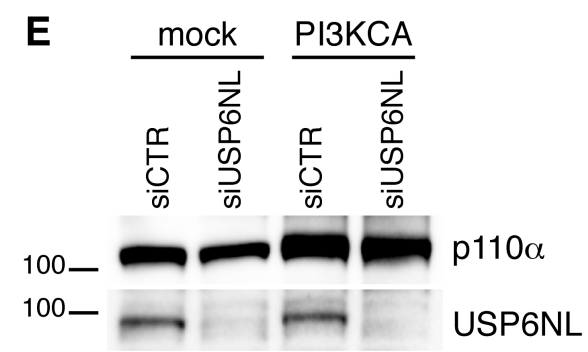

F

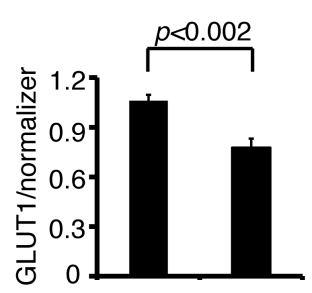

GAPDH
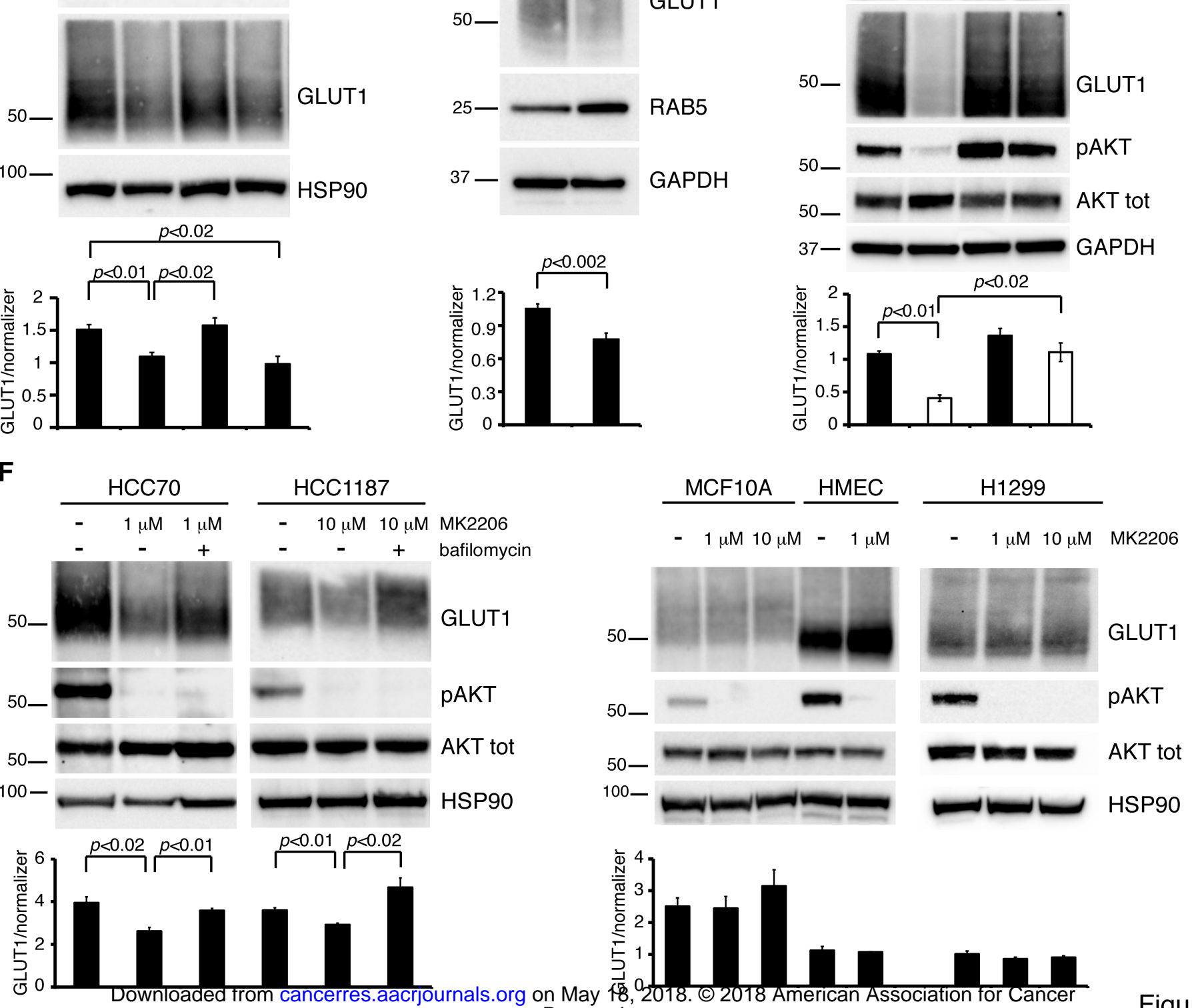
A

CTR
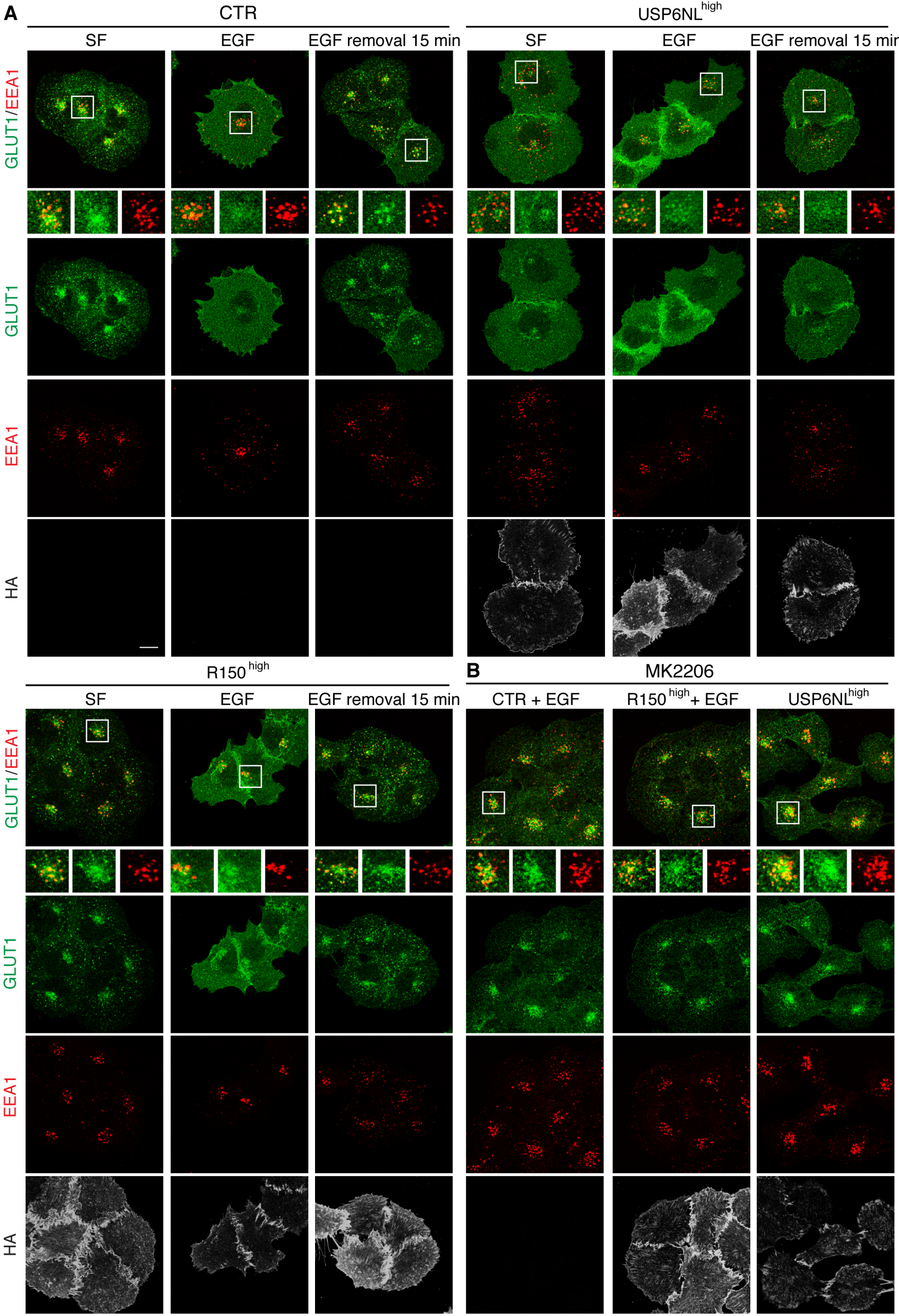

B

MK2206

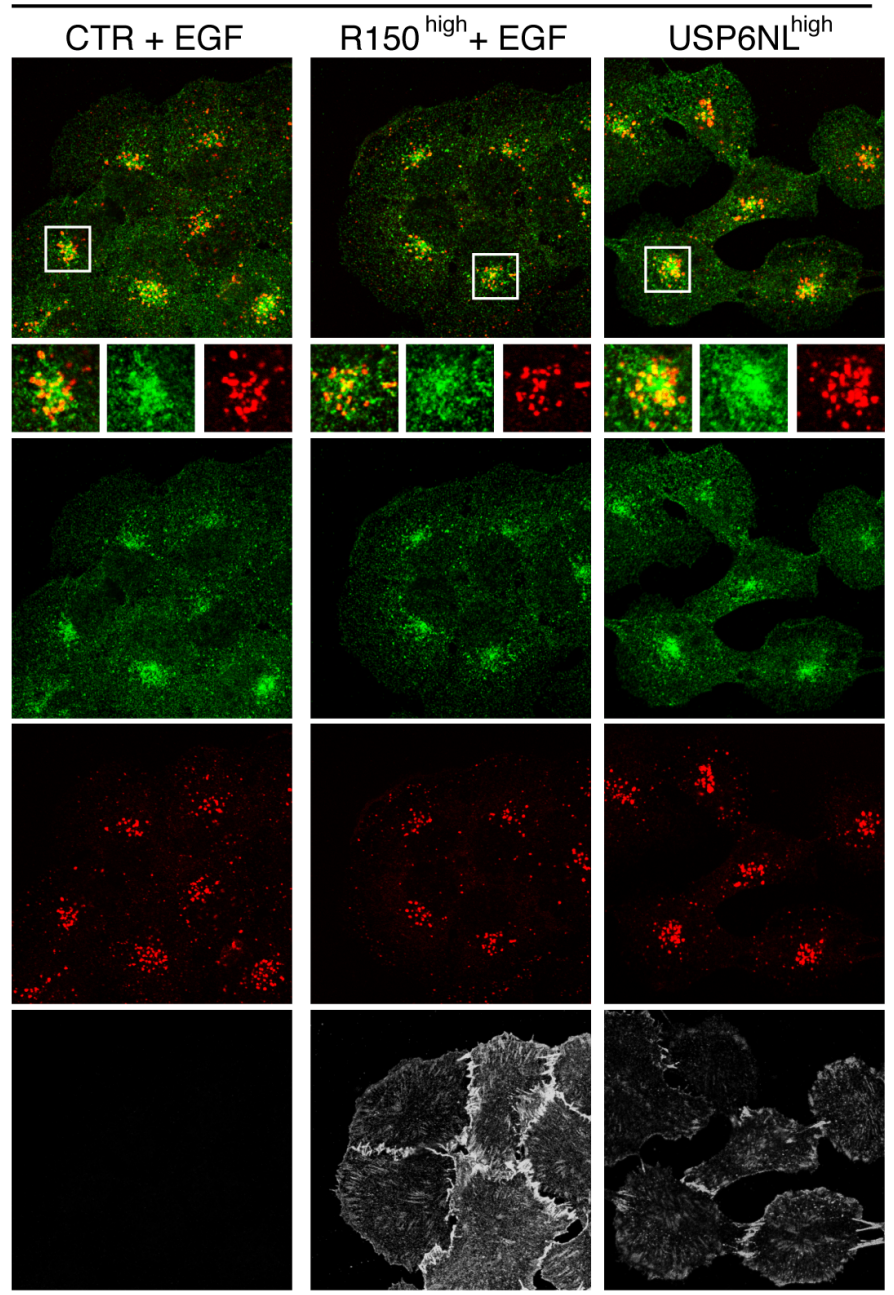




\section{Cancer Research}

ACR American Association

\section{High USP6NL levels in breast cancer sustain chronic AKT phosphorylation and GLUT1 stability fueling aerobic glycolysis}

Daniele Avanzato, Emanuela Pupo, Nadia Ducano, et al.

Cancer Res Published OnlineFirst April 24, 2018.

Updated version

Supplementary Material

Author Manuscript
Access the most recent version of this article at: doi:10.1158/0008-5472.CAN-17-3018

Access the most recent supplemental material at: http://cancerres.aacrjournals.org/content/suppl/2018/04/24/0008-5472.CAN-17-3018.DC1

Author manuscripts have been peer reviewed and accepted for publication but have not yet been edited.

E-mail alerts Sign up to receive free email-alerts related to this article or journal.

Reprints and Subscriptions

Permissions
To order reprints of this article or to subscribe to the journal, contact the AACR Publications Department at pubs@aacr.org.

To request permission to re-use all or part of this article, use this link http://cancerres. aacrjournals.org/content/early/2018/04/24/0008-5472.CAN-17-3018.

Click on "Request Permissions" which will take you to the Copyright Clearance Center's (CCC) Rightslink site. 